\title{
Real-Time Monitoring Concepts for Arterials Using Re-Identification and High-Resolution Data
}
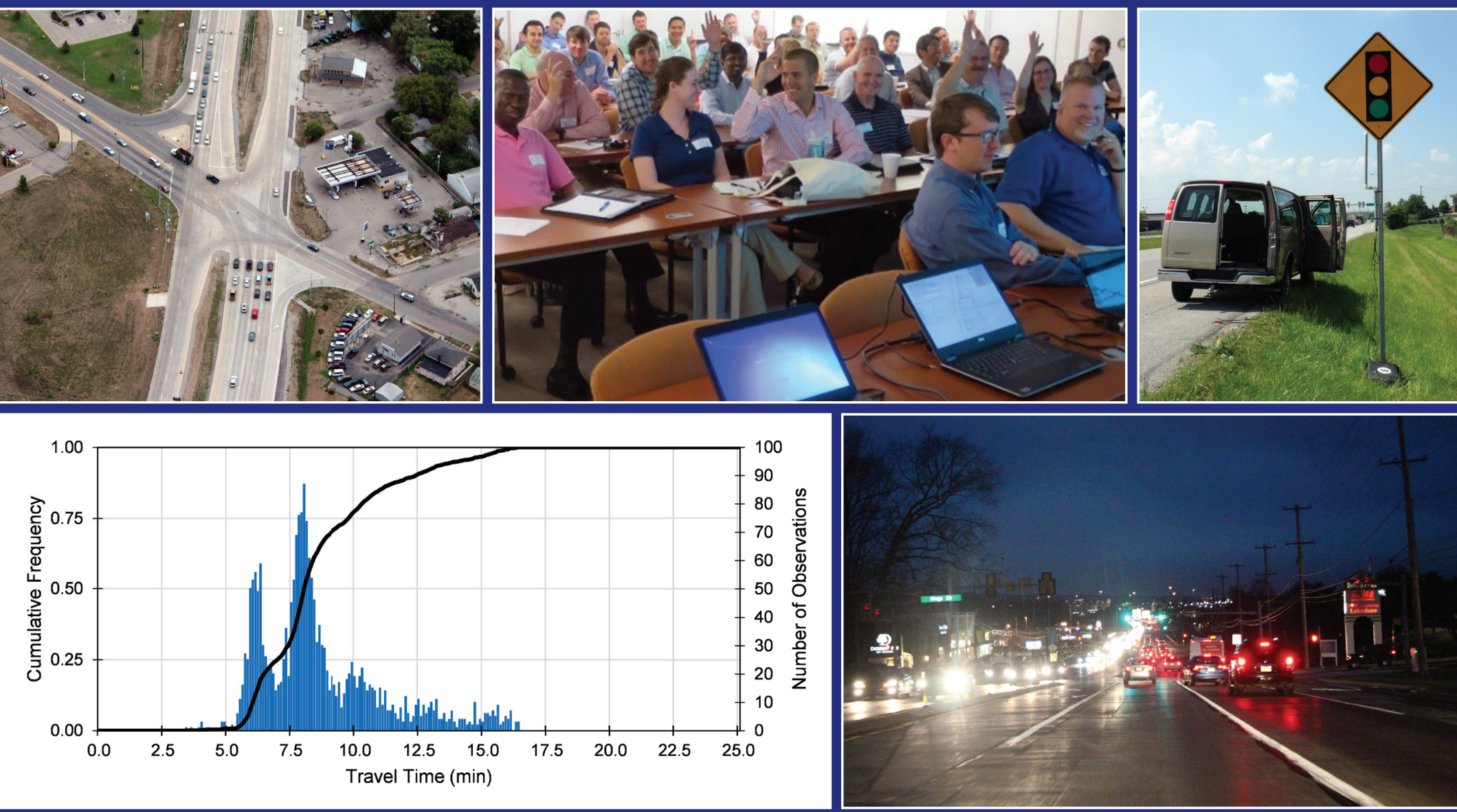

Stanley E. Young, Dennis So Ting Fong 


\title{
Real-Time Monitoring Concepts for Arterials Using Re-Identification and High-Resolution Data
}

\author{
Stanley E. Young \\ Traffax, Inc. \\ Dennis So Ting Fong \\ Traffax, Inc.
}

\section{SBIR Phase 3 Joint Transportation Research Project}

Traffax, Inc.

Purdue University

May 4, 2015

\begin{tabular}{|l|l|}
\hline Deliverable Reference: & D1.4 Real-Time Monitoring Report \\
\hline Project Name: & $\begin{array}{l}\text { Sensor Fusion and MOE Development for Off-Line } \\
\text { Traffic Analysis of Real Time Data }\end{array}$ \\
\hline Contractor: & Traffax Inc \\
\hline Contract Number: & DTFH61-14-C-00035 \\
\hline Contract Term Start & $9 / 4 / 2014$ \\
\hline Contract Term End & $9 / 4 / 2017$ \\
\hline Key Personnel & Stan Young, Darcy Bullock, Dennis So Ting Fong \\
\hline
\end{tabular}




\section{Recommended Citation}

Young, S. E., and D. S. T. Fong. Real-Time Monitoring Concepts for Arterials Using Re-Identification and High-Resolution Data. Purdue University, West Lafayette, Indiana, 2017. https://doi.org/10.5703/12882 84316559

\section{Acknowledgments}

This work was supported by Traffax/USDOT SBIR DTFH6114C00035. The contents of this paper reflect the views of the authors, who are responsible for the facts and the accuracy of the data presented herein, and do not necessarily reflect the official views or policies of the sponsoring organizations. These contents do not constitute a standard, specification, or regulation. 


\section{Table of Contents}

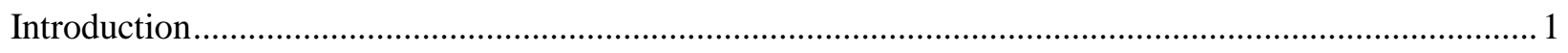

Chapter 1: Applications of Real-Time Arterial Performance Measures .............................................. 4

$1.1 \quad$ Need for Real-Time Arterial Performance Measures …..................................................... 4

$1.2 \quad$ Examples of Real-time Arterial Performance Measures........................................................ 4

Chapter 2: Real-time Visualization of Arterial Travel Time Data.......................................................... 7

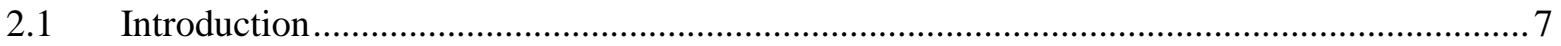

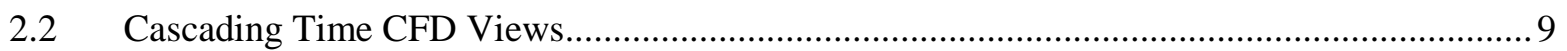

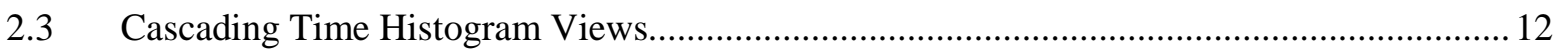

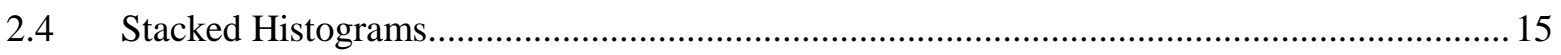

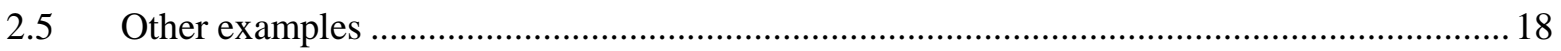

Chapter 3: Technical Considerations for Real-Time Arterial Performance Measures ........................... 26

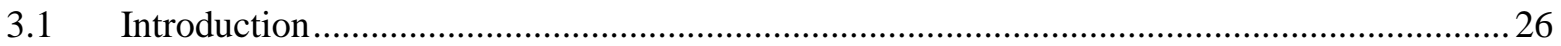

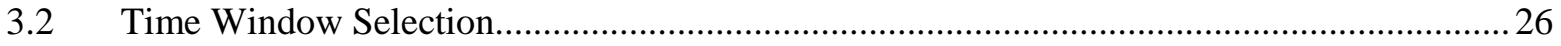

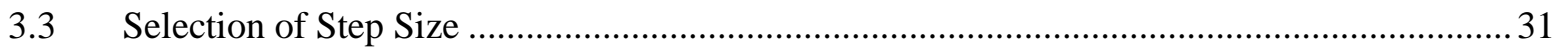

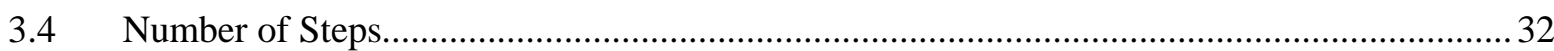

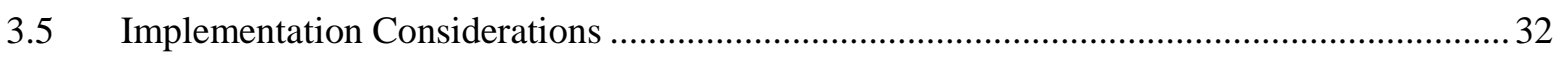

Chapter 4: Real-time High Resolution Controller Data ............................................................................ 33 


\section{Introduction}

This report explores appropriate real-time monitoring methodologies for signalized arterials. The definitions, visualizations and practices identified in the deliverable entitled, "Performance Measures of Interrupted-Flow Roadways using Re-Identification and Signal Controller Data”, were presented from a management perspective in which data is analyzed periodically to assess performance yearly, monthly, or perhaps daily. In this report re-identification data and high-resolution data are considered for real-time monitoring, meaning applications whose time responsivity is measured anywhere from one minute to one hour. Definitions, visualizations, and practices are discussed and extended for such real-time applications that require monitoring of arterial facilities. The document addresses key technical issues related to determining sufficiently long time windows from which to confidently assess current travel-time trends, filtering techniques to identify and remove outliers (in real-time), and methods to assess confidence in the results. Graphical techniques such as Cumulative Frequency Diagrams (CFDs) which were formerly illustrated with archive data for periodic reports are revisited with real-time situational awareness in mind. Evidence suggests that graphical methods similar to CFDs could be implemented in a real-time basis to observe and monitor the status of vehicle flow in an operational context.

Whereas most arterial performance work entails assessment of recorded data, the operations community is seeking operational awareness of the status and performance of the arterial network to react accordingly, particularly assessing the impact on arterial networks that adjoin a freeway whenever incidents or severe congestion on the freeway system force traffic to seek alternate routes. Traffic management centers which originated with primarily a freeway focus are now extending their reach to major arterials, with the goal of proactively diverting traffic to the arterial system when needed. Based on current conditions, the TMC could possibly change the active signal timing plan to one that better accommodates diverted traffic, and monitor the impact of the timing plan. The growing demand for data and tools to actively manage both freeway and arterial networks requires real-time awareness of arterial traffic performance.

At the same time, the operations community is realizing that the sensors and performance measures used for real-time freeway monitoring do not work well on signalized arterials.

- The need for performance measures specific to arterials arose from author's experience with validating real-time outsourced probe traffic data on signal controlled corridors. The methods and statistical framework developed for assessing the fidelity of outsourced probe data on freeways does not work well on arterials due to fundamental differences in the nature of interrupted vehicular flow on arterials.

- Up until about 10 years ago, most roadway traffic monitoring was speed based using loop-based or other point-speed sensor technologies. Freeway operations transitioned from spot-speed based monitoring to a travel time framework in response to the need to provide drivers with anticipated travel time. Although speed sensors are still utilized, they are viewed as an indication of rate of travel time progression for short segments. To obtain reasonable travel time estimates from speed sensors on freeways, detectors should be placed no more the $1 / 2$ mile apart, and preferred spacing for accurate estimation of travel time is $1 / 4$ mile or less.

- Spot-speed measures on arterials were recognized as inherently problematic due to the stop and go and queuing nature of arterial traffic progression. Whereas periodic speed measurements on freeways provides sufficient data to determine the quality of traffic progression, an arterial's 
throughput efficiency is determined by the total number of stops and extent of wait times at signalized intersections, both of which speed measurements fail at determining. For these reasons, direct measurement of travel time is more appropriate for arterials. Floating car runs have been used to measure signal timing effectiveness for decades - an acknowledgement of this principal.

Even when using direct samples of travel time on arterials, the pulsed flow induced by signal systems has the capacity to create complex travel time patterns, requiring a larger number of samples than freeways. One portion of the traffic may progress through the intersection during a green signal, while another portion of traffic is stopped by a red signal and must wait an additional cycle, resulting in a bi-modal travel time distribution. Such complex travel time patterns are captured well in overlay plots and Cumulative Frequency Diagrams. Similarly, these complex stop-and-go patterns on arterials, have to this point, prevented outsourced probe data providers from delivering quality, actionable information on signal controlled roadways. Appropriately capturing the traffic information in real-time based on travel time data from re-identification sources, and the accompanying visualizations, is the main subject of this report. The complex nature of the underlying pattern of travel times makes real-time processing more challenging with sampled data. Technical guidelines such as determining the appropriate (or minimum) number of samples needed for an accurate traffic profile, and the corresponding appropriate time windows of reporting are critical issues to using re-identification data effectively for real-time applications. In addition to real-time progression quality, this report also examines aspects of highresolution controller data that contribute to real-time situational awareness.

\section{Organization of Report}

\section{Chapter 1. Applications of Real-Time Arterial Performance Measures}

This chapter briefly discusses the application of real-time monitoring of traffic progression on arterials within the operations community. Those applications dictate the overall system requirements (in terms of accuracy and observability) that are required from the base data, as well as time and complexity constraints inherent within the operations context.

\section{Chapter 2. Real-time Visualization of Arterial Travel Time Data}

The complex nature of arterial travel time due to signalization presents unique challenges to convey in a concise and meaningful format. Based on Bluetooth re-identification data sets collected extensively over the past several years, candidate visualizations are presented that illustrate methods to concisely portray the travel time distribution, contrasting it against nominal conditions as well as portraying trending as a result of significant demand changes. The visualizations specifically target cascading sequences of CFDs, and histograms. The extensive archive of Bluetooth re-identification data provides many examples of complex bi-modal flow that arises from over-saturation. These visualizations are also applied to outsourced probe data, both to demonstrate the adaptability of the methods to other forms of travel time data, and to compare fidelity with Bluetooth re-identification under similar flow patterns. 


\section{Chapter 3. Technical Considerations for Real-Time Arterial Performance Measures}

Chapter 3 provides guidelines for time aggregation of data for real-time display. These guidelines take into account the cyclic nature of signal control, data sufficiency concerns, and needs for application responsiveness. The guidelines cover appropriately sized time windows, and time steps.

\section{Chapter 4. Real-time High Resolution Data}

Whereas re-identification data provides direct feedback on the travel time which drivers experience in a corridor, high-resolution data provides direct feedback of the performance of individual signals, as well as individual movements on each approach. These are discussed in Chapter 4 with respect to the sample scenario of real-time arterial operations as discussed in Chapter 1. 


\section{Chapter 1: Applications of Real-Time Arterial Performance Measures}

\subsection{Need for Real-Time Arterial Performance Measures}

Applications of real-time arterial performance monitoring within this report address the concerns of the traffic operations community by seeking to extend applications such as incident management and traveler information to the primary arterial network. Specifically of interest are the arterial routes that intersect with the freeway system, and provide alternative routes for travelers as a result of a freeway incident. Real-time monitoring and performance assessment extend from the daily, weekly, monthly and yearly time aggregations used for system level management to those aggregations needed for ongoing situational awareness. These are typically time aggregations of the latest one minute, five minute, 15 minute and hourly performance criteria. Such time responsiveness is needed to support management decisions such as whether or not to divert traffic in response to accidents or major congestion events, or to change signal timing plans to handle diverted traffic.

Operations, within the context of intelligent transportation system (ITS) and traffic management centers, are typically reactionary in the case of unexpected or unusual perturbations on the roadway network. This includes crashes and weather phenomenon that disturb normal operating patterns. Even special events such as large sporting events, though not unexpected, do produce unusual network impacts that are typically not planned. Traffic management centers (TMCs), which originated primarily for the management of freeways, are responsible for the coordination of emergency response personnel, maintaining traffic flow in the vicinity of an incident, and doing as much as possible to ensure that congestion due to incidents is mitigated efficiently. This paper considers real-time arterial performance measures from the viewpoint of extending those responsibilities to managing the impact on adjacent arterial networks as a result of incidents that impact the freeway network.

Real-time behavior is already built into many signal control systems for signal actuation. This includes such behavior as servicing side streets with detector presence activated calls, pre-emption or priority control for emergency or transit vehicles, and adaptive control to adjust in real-time to fluctuations in demand. Such real-time behavior is typically intended for optimizing signal efficiency within the ranges of anticipated demand under normal operating conditions, and carried out by algorithms within the signal controller. In contrast, real-time performance monitoring required by TMCs during major incidents that impact the arterial network encompasses measures of delay and other impacts for use by personnel (not signal controllers) to respond appropriately as a result of non-normal operating conditions. Such responses may include posting traveler information, dispatching personnel to direct traffic, or setting up alternate routes and detours.

\subsection{Examples of Real-time Arterial Performance Measures}

Following is a scenario depicting a traffic management center need for arterial performance information during a major traffic incident. Figure 1.1 depicts a section of Interstate 70 outside the Baltimore beltway to the west of the city. A red star indicates the location of a crash on westbound I-70 of sufficient severity that it will diminish the capacity of I-70 to handle westbound demand for an anticipated period of two hours or longer. Such a scenario is indicative of an incident with major injuries or fatalities requiring emergency medical personnel. Diversion routes westbound on I-70 include US-40 to the south and Rogers Avenue to the north. US40 is a multi-lane major arterial accessible via the US29 interchange that forms an approximate four mile detour around the incident, depicted in a dashed green line in the Figure. 
Rogers Avenue also parallels I-70 between the interchange of US29 and the intersection of MD32. It is a two-lane facility, typically with stop-controlled intersections, that provides an approximate seven mile bypass to the north of the highway. It is depicted in a dashed blue line in the Figure.

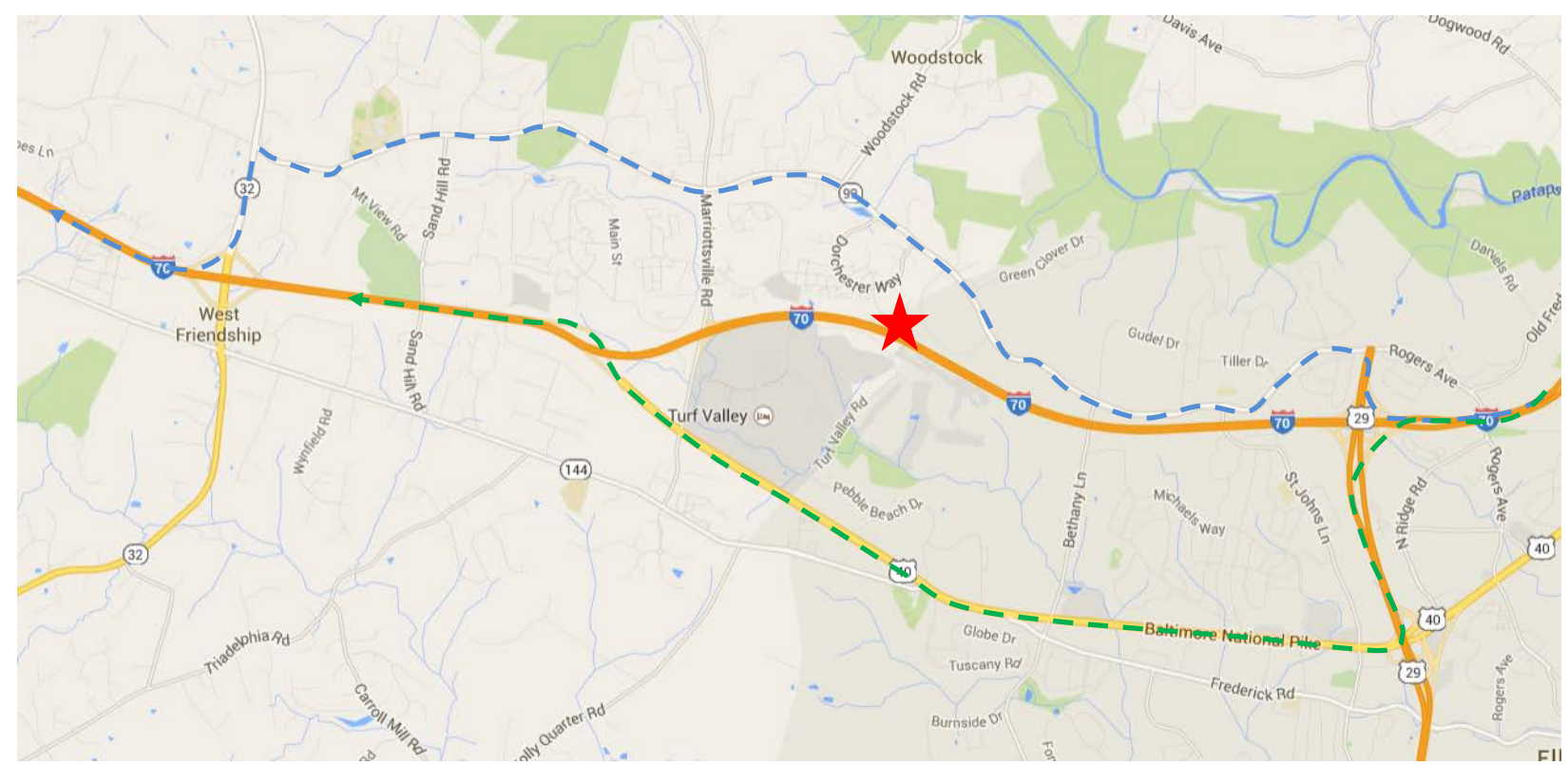

Figure 1.1 Arterial diversion paths as a result of freeway incident on I-70 west of Baltimore

The traffic management center is charged with managing incident response, providing appropriate traveler information, and managing traffic through the duration of the incident. The discharge of such duties on the freeway network is well-defined as a result of two or more decades of practice, and ever increasing technical capabilities of traffic monitoring available on our freeway system. This scenario exemplifies the need for real-time arterial performance data because the only traffic diversion alternatives involve the local arterial system. Existing tools and data sources inform the process for the freeway network including outsourced probe data for speed and travel time, incident reporting and response timelines based on emergency responder reports and associated traffic data, and other tools that have been refined to provide metrics to assess the incident and inform the TMC. These tools assist the TMC in vectoring appropriate field personnel and providing accurate traveler information. However, currently this is practiced primarily on the freeway network. Supporting systems that provide needed performance metrics for assessing the impact to the diversion route (arterials) is for the most part absent. Information is needed by TMCs to answer questions, such as the following, related to knowledge of arterial diversion routes:

With respect to Incident Management

o What is the most direct route, based on current traffic conditions, for emergency services to access and egress from the incident scene?

o Is there sufficient reserve capacity available on immediate diversion routes to direct traffic or set up a detour?

o Should incident managers be "metering" the number of vehicles being diverted (i.e. detouring a regulated number of vehicles at a time so as not to oversaturate diversion routes)? 
With respect to Situational Awareness and Active Traffic Management

o What impact is the diverted traffic making to normal traffic patterns on the local arterials?

o Is an adjustment to signal timing warranted, and if so is the new timing plan effective at handling the diverted traffic as well as normal arterial corridor demand?

o Should police be dispatched to non-signalized intersections (such as Rogers Avenue) on diversion routes for traffic control?

o Is one diversion route better than another, or will one have less impact to local traffic than another?

o Should multiple, simultaneous detours be implemented?

o What are the impacts on perpendicular routes (MD 32, Marriottsville Road, Saint John's Lane, US 29) that intersect the prime detour routes? Are side street delays becoming excessive if more green time is allocated to handle the diverted traffic?

o How long after the detour is in place until the alternate route begins approaching unacceptable performance, (Level of Service F based on Highway Capacity Manual standards)?

o How long after the I-70 issue is resolved does traffic take to return to "normal" conditions?

$>$ With respect to Traveler Information

0 What is the anticipated travel time on each diversion route?

o What message/s should be delivered to drivers on changeable message signs and local media upstream of the incident?

o What information should be told to travelers that typically use the diversion routes or routes perpendicular to the diversion routes (MD 32, US 29, etc.) concerning the incident or diversion?

o Can we inform motorists/travelers how long it will take until traffic is restored to "normal" conditions, both on I-70 and the diversion route(s)?

With respect to after action reports and briefing

o What was the experienced travel time for those that diverted, versus those that remained on I-70?

o What was the impact to local traffic on US-40 and/or Rogers Avenue as a result of diverted traffic?

o What was the impact of the traveler information? How many people diverted in response to the information?

o Should an enforced diversion have been used in lieu of a volunteer diversion in response to traveler information?

o Were alternate signal timing plans sufficient to handle diversions, are better plans needed?

o Was capacity on the diversion route(s) utilized to their maximum extent to mitigate the impact of the incident?

o Was capacity sufficient/appropriate on diversion routes for traffic mix (height, weight, width, and turning restrictions)?

These and other questions and concerns which arise with respect to real-time traffic management during incidents that impact arterial networks are the purview of this discussion. The treatment of arterial performance measures is pushed to the perspective of appropriate point-in-time traffic impact analysis, providing the best operating picture with which to make management decisions in the areas of incident management, traffic management, and traveler information. 


\section{Chapter 2: Real-time Visualization of Arterial Travel Time Data}

\subsection{Introduction}

The complex nature of arterial travel time due to the impact of signalization presents unique challenges to display in a concise and meaningful format for real-time operations. This chapter presents several methods adapted from methods originally developed for archive (non-real-time) data that support traditional arterial performance measures. These traditional methods are based on Cumulative Frequency Diagrams, and histograms, adjusted to display point-in-time visualizations. The methods attempt to provide a compact display, highlighting the change in current conditions over time (such as the last 5, 10, 15 minutes), and contrast current travel time conditions with nominal conditions for the time of day and day of week. These visualizations employ cascading portrayals of travel time data to convey the dynamic changes in traffic patterns as they unfold. Archive Bluetooth re-identification data sets collected extensively as part of the I-95 Corridor Coalition's Vehicle Probe Project validation process are used as the basis to illustrate the methods. This Bluetooth data archive provides many examples of complex bimodal flow that arise from over-saturation on signalized roadways. Such conditions are anticipated when traffic diverts onto the arterial network as a result of a major incident on the adjoining freeway network. This is the operations scenario of primary interest voiced frequently from traffic management center personnel. In addition to the Bluetooth re-identification data archive, the visualization methods are also demonstrated on outsourced probe data to demonstrate the flexibility of the process, as well as inform the reader about the contrast of data fidelity available from each data source.

Traffic conditions from an approximate 1.0 mile long section of US-1 in New Jersey are used to illustrate the construction of the proposed visualizations. This section of US-1 begins at Washington Road and proceeds north to the intersection with Scudders Mill Road. Data was collected from September 10 through September 26, 2013. US-1 in this location consists of three plus lanes of traffic in each direction, with two signalized intersections.

Twenty-four hour weekday overlay plots for both Bluetooth and outsourced probe data are shown in high resolution in Figure 2.1. Bluetooth re-identification data is shown in blue in the top graph, and a sample of outsourced probe data from the I-95 Vehicle Probe Project (VPP) is shown in red in the bottom graph. Figure 2.2 contrasts the travel time data using an overlay scatter plot on the left and CFD plots to the right. The Bluetooth data in these overlay and CFD plots reflect a significant AM and PM peak hour delay, as well as a bi-modal platoon progression. Vehicles entering this section of roadway have about a $50 \%$ probability of progressing through green at both signals, and are equally likely to be stopped and required to wait for the next cycle at one of the two signals. During peak periods, particularly the PM peak period, more of the platoon is forced to wait until the next cycle, and very few vehicles are able to progress through both signals on green. The AM peak period has similar properties as the PM peak period, but the transition between the faster and the slower modes occurs over a period of about one hour, and a larger percentage of the traffic stream is still able to progress through on green even during the peak AM period. It is this AM transition period that is used to demonstrate some of the real-time visualizations of traffic. 

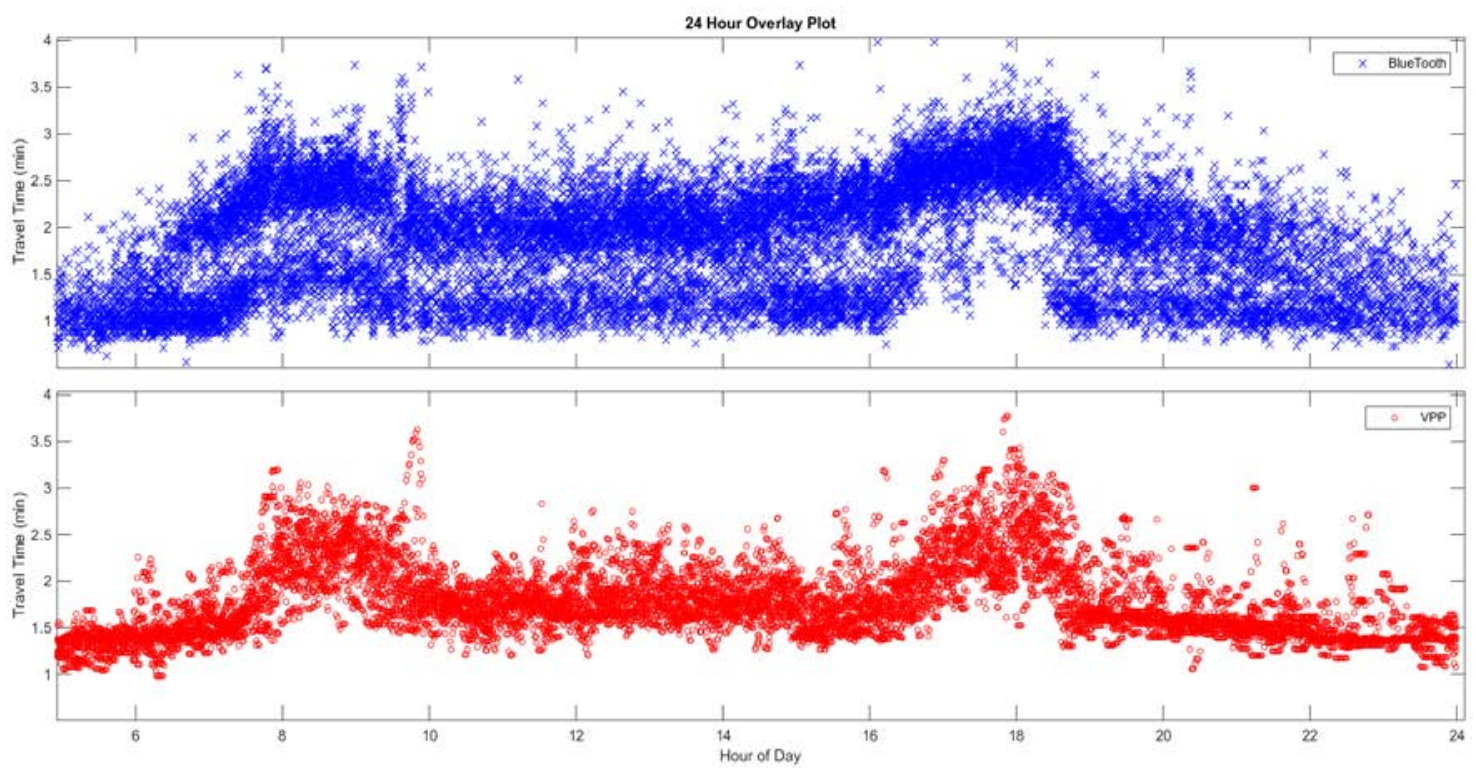

Figure 2.1 24-hour weekday overlay plots of sample data for US-1 data in NJ
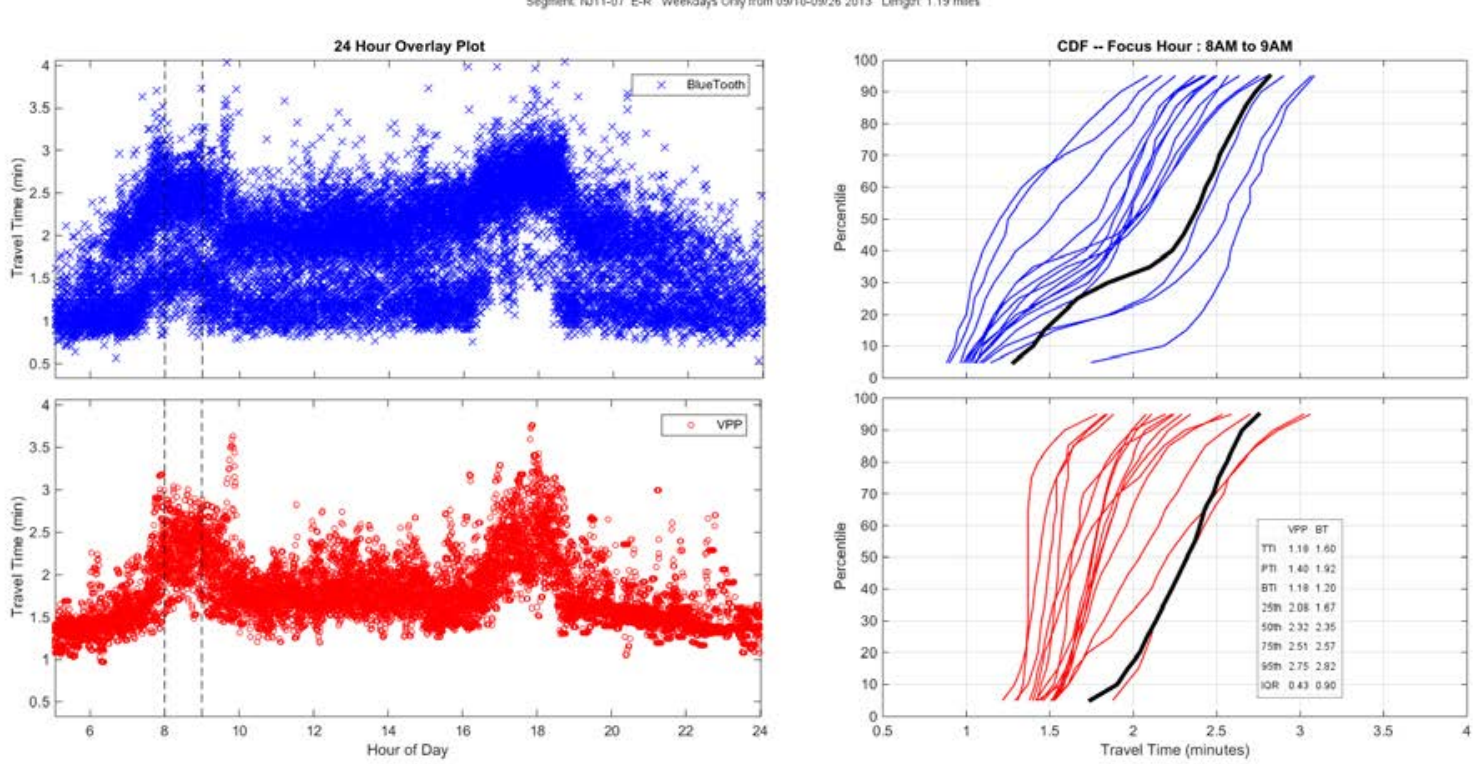

Figure 2.2 Sample Re-identification data used for illustrating real-time visualizations from US-1 in NJ 
Twenty four-hour overlay data, and corresponding CFDs provide an effective basis for arterial performance measures and visualizations. The data from this US-1 case study, particularly during the escalation of the AM peak period as shown in Figure 2.1, is used as the basis for construction of real-time visualizations. The methods are also applied to the outsourced probe data (shown in red) to demonstrate that such methods are adaptable for any travel time source. The I-95 VPP Arterial Validation Report of 2015, available from the Coalition's web site provides a full assessment of outsourced probe data fidelity. Based on this report outsourced probe data is recommended only on signalized corridors with signal densities of less than one per mile. US-1 meets this criterion. Even though this specific segment has two signals in a one mile extent, the overall corridor in this vicinity averages signal spacing of less than one per mile. Though the outsourced probe data is of less fidelity, the proposed visualizations are equally adaptable to both Bluetooth and outsourced probe data. The accuracy of the visualizations is limited only by the fidelity of the base data.

Three separate visualizations are constructed and demonstrated using the US- 1 data above. The US-1 data from Figure 2.1 is treated as if it comes from a single day, though it is a multi-day overlay taken from an approximately two week period. This approach effectively increases the data density by a factor of ten, resolving any concerns related to sufficiency of the number of travel time data samples. Such concerns and associated guidelines are a significant issue with re-identification data addressed in the next chapter.

At the end of this chapter, the proposed visualizations are applied to another roadway that exhibits complex travel time patterns similar to US-1, but is not an extremely high-volume roadway as is US-1 in New Jersey.

\subsection{Cascading Time CFD Views}

The first visualization is similar to the standard cumulative frequency diagrams (CFDs) shown in the right of Figure 2.2, but augmented to emphasize time sequence and trending. CFDs are also referred to as cumulative distribution functions (CDFs) in statistical literature. The two terms are used interchangeably in this context. The real-time visualization using CFDs requires the selection of sequential time windows for display. A basic illustration of the time sequencing process is illustrated in Figure 2.3 in which an approximate one hour window from noon to $1: 00 \mathrm{pm}$ is highlighted. The arrow indicates direction of increasing time. Note that the ensemble of CFD curves in Figure 2.2 is created by dividing the travel time data into one hour segments (creating one hour windows) throughout the day, and then plotting the CFD for each hour. The differences between the historical view shown in Figure 2.2 and the real-time visualizations are the size of the time window, the size of the time step, the number of time steps, and the visualization scheme used to indicate time ordering. In Figure 2.2 (CFD to the right), the size of the data window is one hour, and the size of the time step is also one hour. All one hour time steps are shown throughout the day. There is no visualization scheme in Figure 2.2 to indicate time sequence other than highlighting the focus hour in contrast to the ensemble of other hours. 


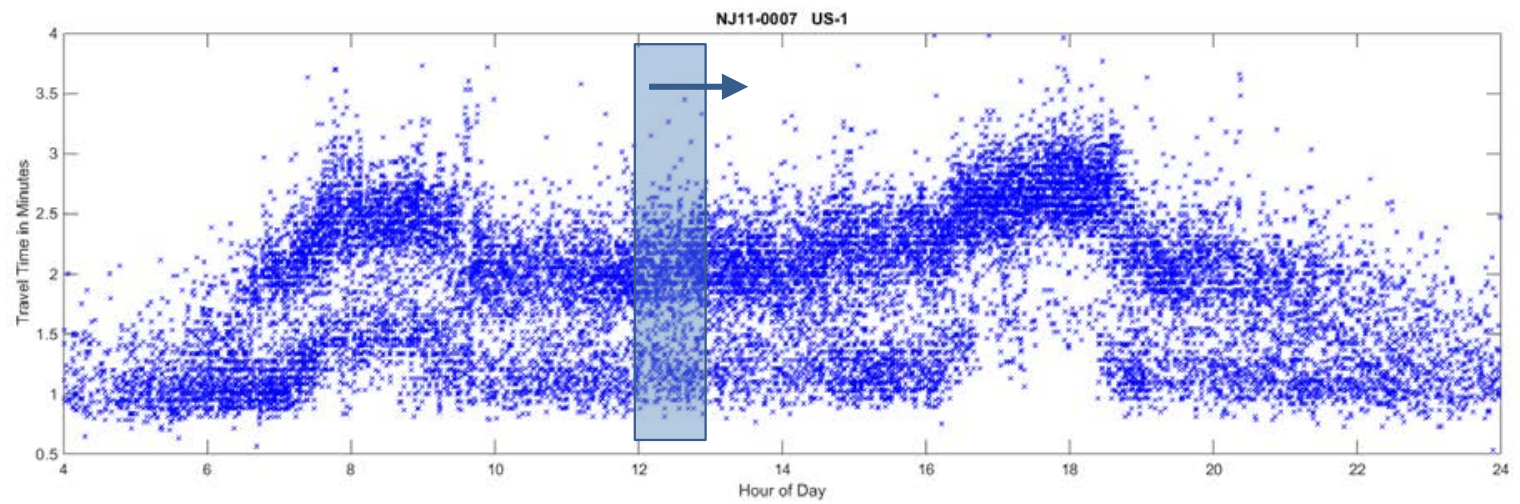

Figure 2.3 Illustration of a sliding time one-hour window for construction of time sequence data

Figure 2.4 is an expanded view of Figure 2.3 showing travel time between the hours of 6:00 am and 9:00 am on US-1 during the escalating traffic of the am peak period. The figure illustrates an alternative time windowing scheme. The time window is set to 15 minutes, and the time step to five minutes. The number of time steps is set to 10, such that the ensemble of CFD curves reflects travel time changes over the past 45 minutes (time step times the number of time steps minus one time step). Each box represents a 15 minute time window staggered five minutes (the time step) from the preceding time window. The most current time window is from 7:30 am to 7:45 am with the corresponding Bluetooth re-identification data highlighted in red. The oldest time window is from 6:45 am to 7:45 am indicated by the shortest rectangular box. Note the heights of the rectangular windows are simply for ease of visual reference in the figure, each represents an equal 15 minute period.

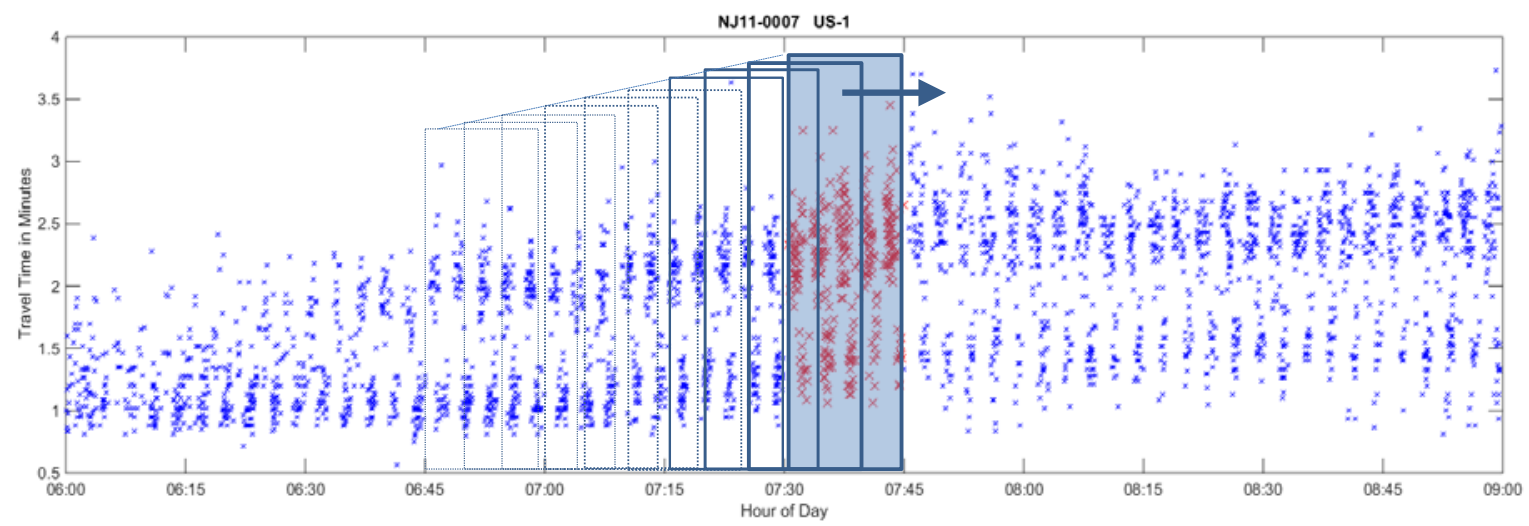

Figure 2.4 Construction of real-time sliding 15-minute time windows from 6:45 am to 7:45 am 
In addition to illustrating the sliding window concept for real-time visualization, Figure 2.4 illustrates two additional aspects of the travel time data that need to be considered for real-time visualizations.

First, the AM peak period that is clearly evident is Figure 2.3 when viewing data for an entire 24 hour period is not as distinct when viewing data from only the 6 am to 9 am time period. Reductions in speed, or conversely increases in travel time, that appear as distinct trends at large time scales are typically gradual increases that are less distinct when viewed at highly granular time scales. As real-time visualizations typically work with granular time scales of 15 minutes to 2 hours, real-time visualizations need to accentuate significant changes in traffic on a minute to minute time frame during major slowdowns.

Secondly, the striations in the re-identification data at this zoom level are quite common. Each striation corresponds to a signal cycle. Because this corridor uses fixed-time signals, the start of the green portion of the cycle phase is consistent from day to day, thus creating repeating green bands when a vehicle can progress through the signals on the corridor. At this level of time granularity, these striation patterns are similar to space-time diagrams, showing when a vehicle can progress through a signalized corridor. The horizontal distance between the striations corresponds to the cycle length. On this corridor the cycle length is approximately three minutes because approximately five cycles are observed in a 15 minute period. The relationship between the cycle length and width of the time window are explored more in chapter 3 when considering minimum and maximum time windows and time steps. Note, striations reflecting cycle lengths are not always visible on 24 hour overlay plots. They appear only on signalized corridors that operate on fixed cycles, or near-fixed cycles. Any signal timing schemes that dither the cycle start times from day to day would not exhibit such striations. However, for any real-time system, the visualization will be constructed on a cycle-by-cycle basis in which these effects will always be present.

The corresponding ensemble of CFD curves for the time windowing scheme exhibited in Figure 2.4 is shown in Figure 2.5. The current fifteen minutes is shown in bold blue line for the 7:45 am instance in time, and previous time steps are shaded in incremental steps according to the color shading map shown to the right. The trend of escalating travel time becomes apparent based on the color and line weighting sequence in accord with the escalating traffic during the morning peak period. The time windows begin at 7:00 am (such that the oldest time window reflects travel times from 6:45 am to 7:00 am), and go to 7:45 am (such that the most recent time window reflects travel time from 7:30 am to 7:45 am). As time increases from 7:00 am to 7:45 am, the CFD curve appears to progress to the right. This is an indication that, as the morning rush hour progresses, traffic is forced to wait longer and longer while traversing this segment. The central tendency of travel time, as indicated by the $50^{\text {th }}$ percentile, increases from 1.3 minutes to 2.2 minutes from the earliest to the latest window. The shift of the 's' shaped curve to the right and down indicates that fewer and fewer vehicles are able to progress through the corridor on green, and increasingly more vehicles are forced to stop and wait until the next cycle at one of the two signalized intersections. 


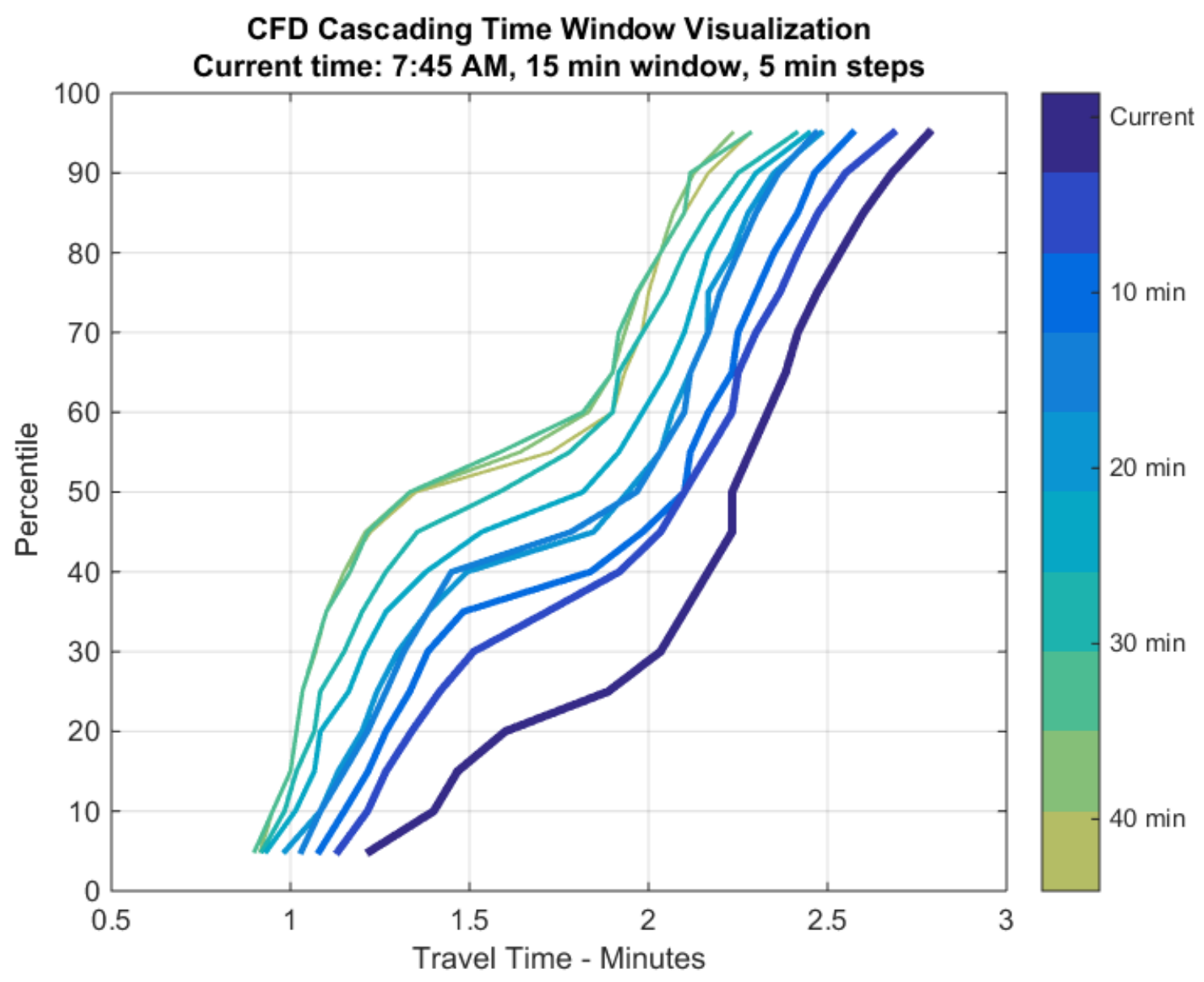

Figure 2.5 Cascading CFD visualization with 15 minute window, 5 minute time-step, and 10 steps

\subsection{Cascading Time Histogram Views}

Similar to CFD views, histograms can also be constructed showing time sequence windows. Note that normalized histogram views, also regarded as probability density function views, or PDFs, are proportional to histograms, with the primary difference being the scale of the y axis. Any discussion on the construction of real-time histograms is also applicable to PDFs. Figure 2.6 is an example of a cascading histogram view of the same traffic situation explored in section 2.2. It displays the same data from US-1 between 7 AM and 7:45 AM using 15 minute windows, 5 minute steps, and 10 steps. Similar to the CFDs, Figure 2.6 uses color and line type scheme to differentiate the time windows, providing a summary of the previous 45 minutes traffic trending in a single glance. Note that although histograms are typically viewed as bar charts, it is difficult to portray more than one bar chart on a single graph. For this reason line graphs are used to portray the cascading sequence of histograms over this 45 minute section. 


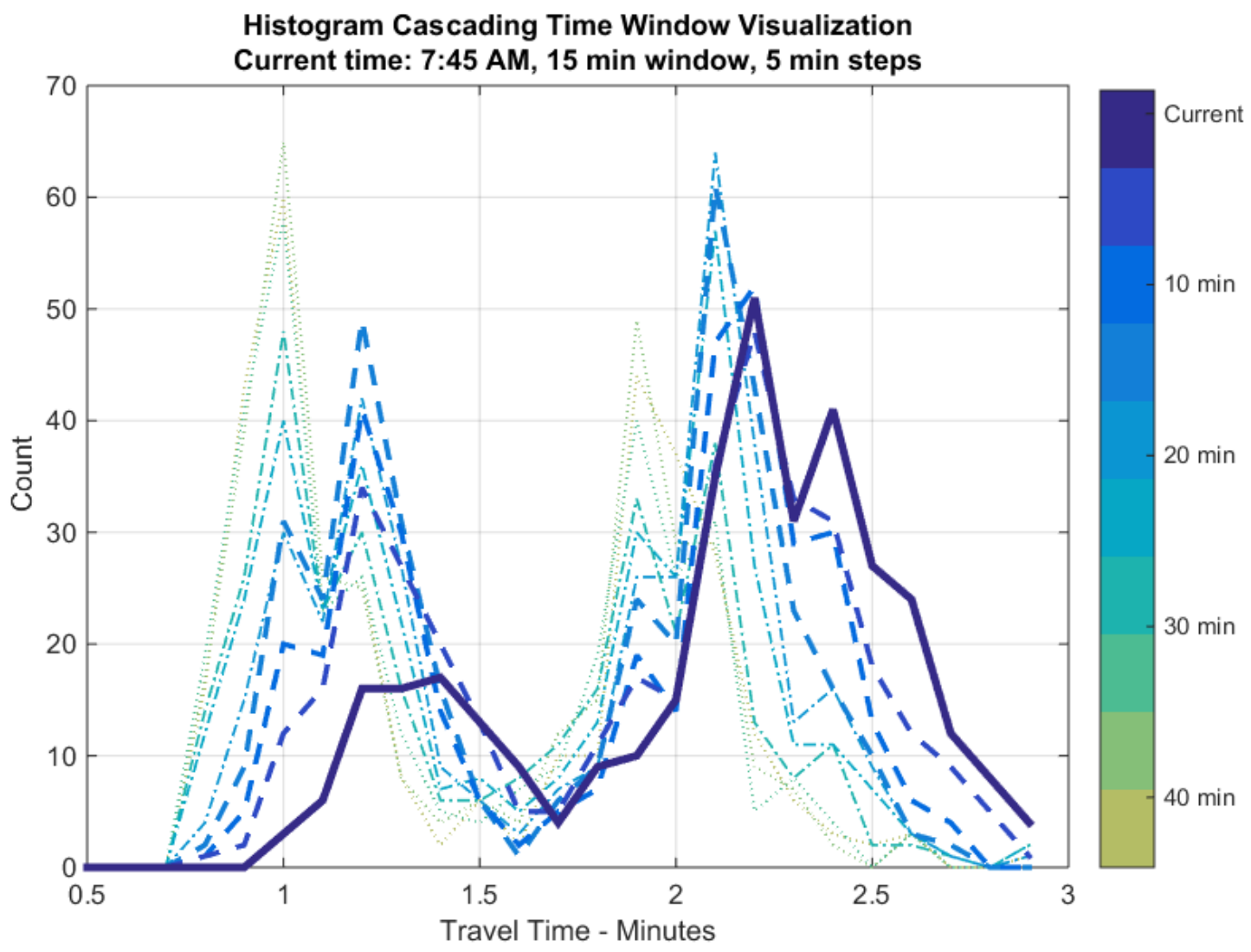

Figure 2.6 Cascading Histogram Visualization: 15 minute window, 5 minute time-step, and 10 steps

The cascading histograms appear to shift to the right in time, similar to the cascading CFDs. The bi-modal distribution is clearly evident, with two distinct travel times for each time window. As the morning peak period progresses, the right peak becomes more dominant (as well as shifts to the right), indicating a large proportion of the traffic stream is delayed by an additional signal cycle.

Histogram views are better at conveying (or perhaps are a more intuitive representation of) the nature of a single travel time distribution than are CFDs, but histograms are not as compact as CFDs. The complexity of multiple histograms on a single chart may counteract any advantages from an intuitive standpoint.

The time steps in a cascading histogram can be staggered for ease of viewing as shown in Figure 2.7. A constant offset is added to the independent variable (y axis) to each time window as it is plotted, accentuating the cascading effect.

Many of these parameters, such as color scale, line types and weights, and whether or not to use vertical offsets for time sequence cascading are user preferences. Options such as these should be considered as user adjustable parameters in real-time visualization systems. 


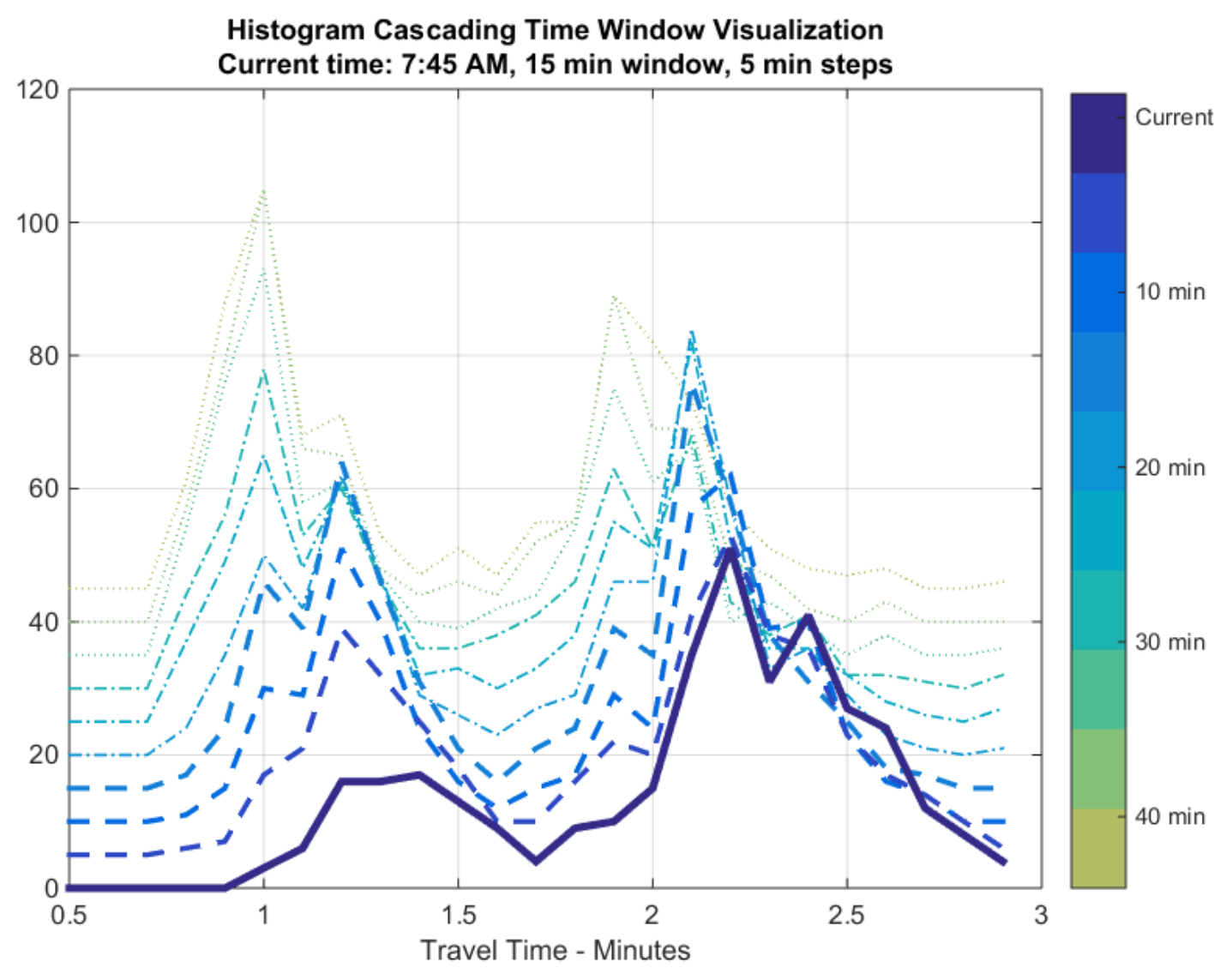

Figure 2.7 Cascading Histogram Visualization accentuated with constant offsets 


\subsection{Stacked Histograms}

Stacked histograms for display of real-time travel time data is a variant on the cascading histogram method. Rather than cascading histogram line plots, a stacked histogram using bar charts is constructed. Each layer of the stacked histogram represents a time window. Unlike the cascading views in which time windows overlap, the stacked histogram sets the time window equal to the time step. For the following two illustrations, the time window is set to five minutes (rather than 15), equal to the time step, for the construction of stacked histograms. Figure 2.8 is a top-stacked histogram reflecting the escalation of traffic on US-1 from 7 am to 7:45 am. The oldest data is on the bottom (in dark yellow). Each time window is stacked on the previous such that the top most layer (in deep blue) is the most recent data. The color gradient on each bar reflects the general shift in traffic as observed in previous visualizations. As new data is added to the graph, the lowest layer is dropped, and a new layer is added. The overall shape of the histogram provides the distribution envelope of the travel times over the 45 minutes of observations. The shift in color reflects the trend.

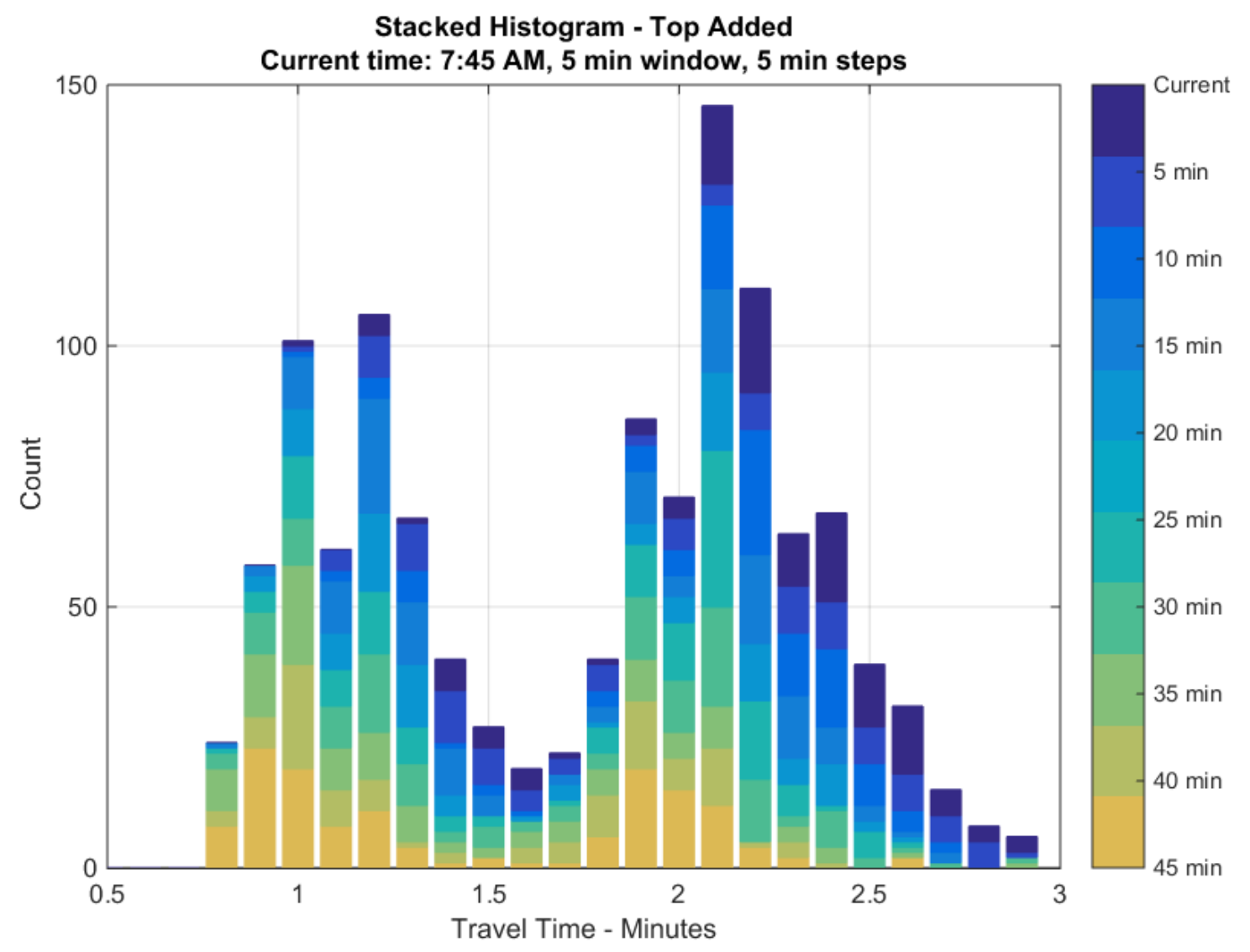

Figure 2.8 Top Stacked Histogram: 5 minute window, 5 minute time-step, and 10 steps 
Figure 2.9 is a bottom stacked histogram, such that new data appears at the base of bar graphs, rather than at the top of bar graph.

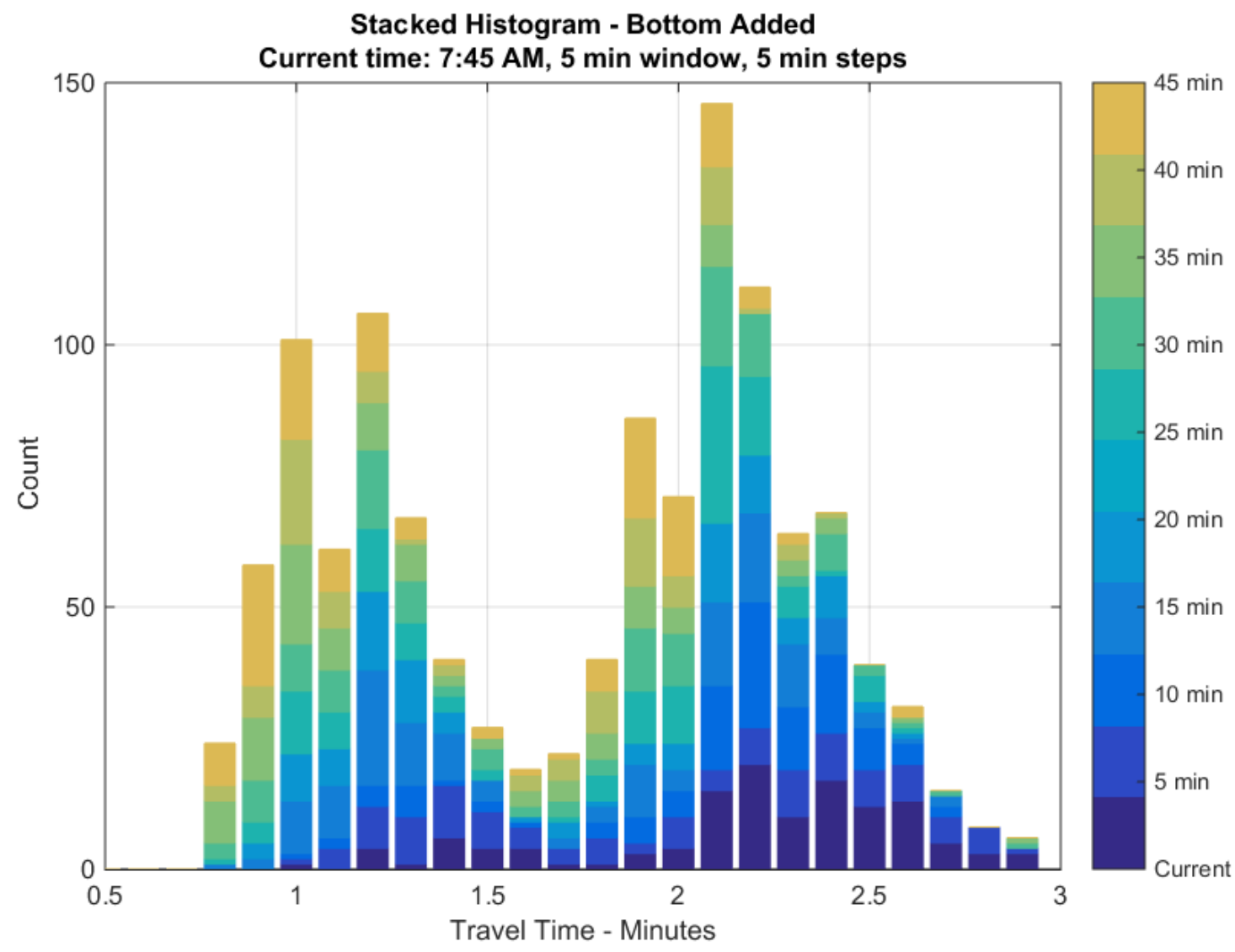

Figure 2.9 Bottom Stacked Histogram: 5 minute window, 5 minute time-step, and 10 steps 
Color selection for stacked histograms may be more effective in a broader range. The top stacked histogram from Figure 2.8 is repeated in Figure 2.10, but using a color map that spans additional hues. The contrast between the most recent data (in blue) and the oldest data (in red) provides additional visual cues of the trend over the observed period.

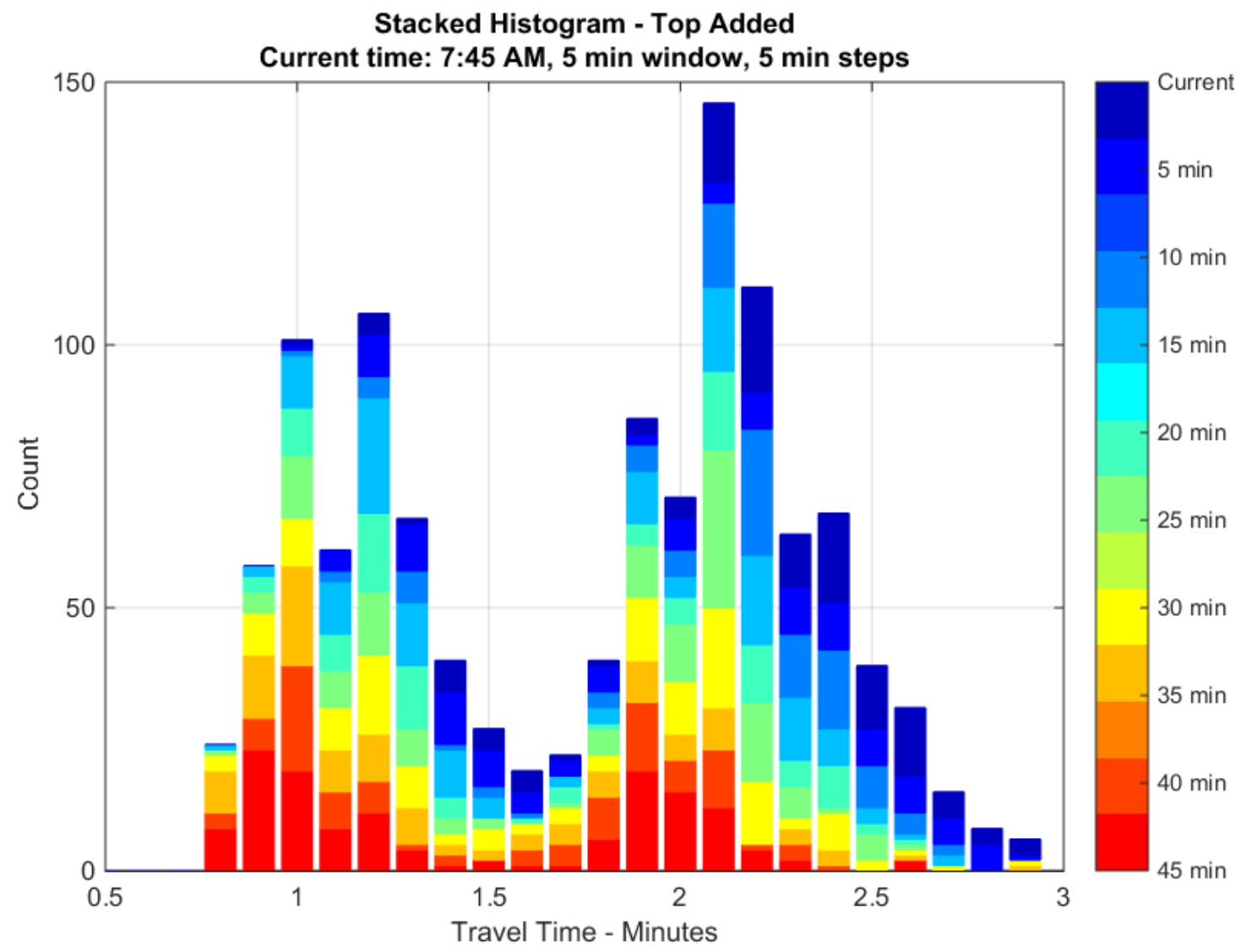

Figure 2.10 Top Stacked Histogram with wider color scheme: 5 minute window, 5 minute time-step, and 10 steps 


\subsection{Other examples}

Additional examples of the basic visualizations are presented for two more case studies. The first is from US-29 in Virginia. The segment begins at the intersection of VA123, also known as Chain Bridge Road, and proceeds west - southwest until it ends at the intersection of Jermantown Road. The corridor is 1.4 miles in length with two lanes of traffic in each direction, has a 35 mph speed limit, and includes seven signalized intersections. Figure 2.11 shows the 24 -hour weekday overlay plot from data collected May $8^{\text {th }}$ through May 19 th 2014 for the hours of 6:00 am to midnight. The evening peak period is depicted in Figure 2.12 from 4:30 pm to 6:30 pm, encompassing the escalation of the evening peak period congestion. This time windowing sequence is shown in Figure 2.12, with 15 minute windows stepped at 5 minutes beginning at 5:45 pm, stepping backwards to 5:00 pm with each window encompassing the previous 15 minutes. As with the initial example, the congestion in the two-hour time period plot is not as prominent as it is in the full day view. Regular striations associated with fixed-time signal operations are also evident.

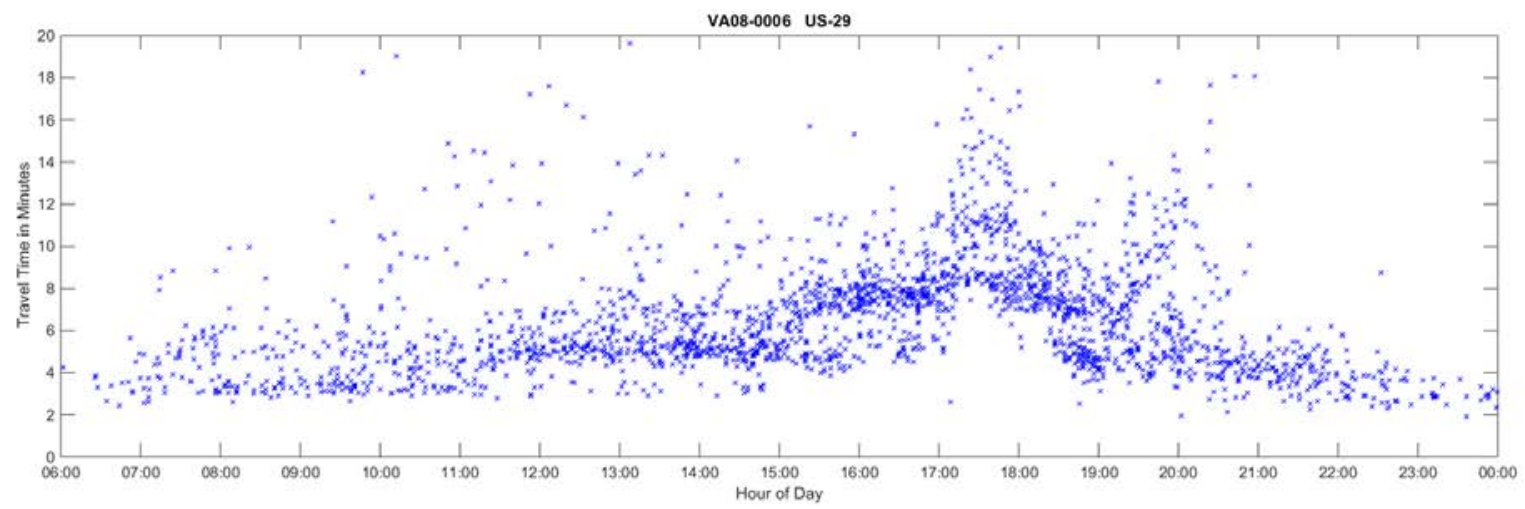

Figure 2.11 24-hour overlay plot for data from US-29 in Virginia

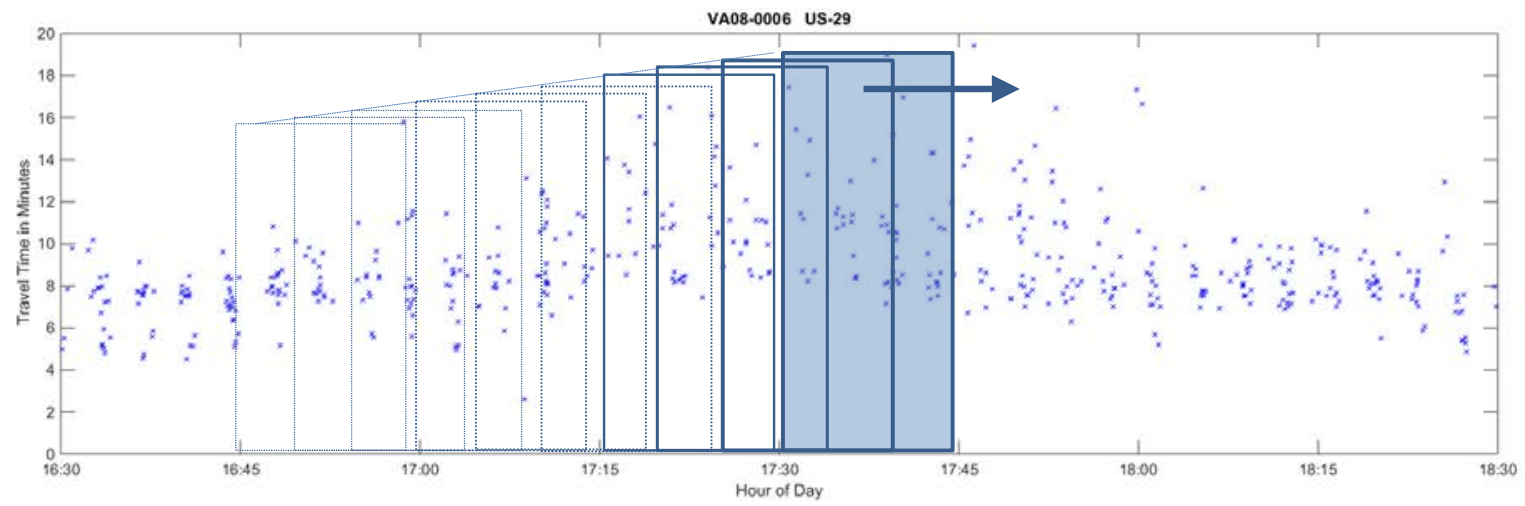

Figure 2.12 Overlay plot of US-29 from 4:30 pm to 5:30 pm with windowing sequence highlighted 
Figure 2.13 is the cascading CFD visualization of this time period. The diagram indicates that the travel time began escalating about 5:15, and plateaued about 5:30. The three oldest CFD curves appear to coincide, the next three shift to the right, indicating escalating travel time. The remaining, most current four travel time windows also coincide, indicating that the travel time has plateaued.

Figure 2.14 shows the cascading histogram and cascading stepped histogram of the same time period respectively. The change in travel time patterns is observed in the relative size of the two primary modes. The histograms do not appear to shift to the right (as in the example from US-1 in New Jersey), but rather the relative size of the two modes changes.

Top and bottom added stacked histogram visualizations for US-29 are shown in Figure 2.15.

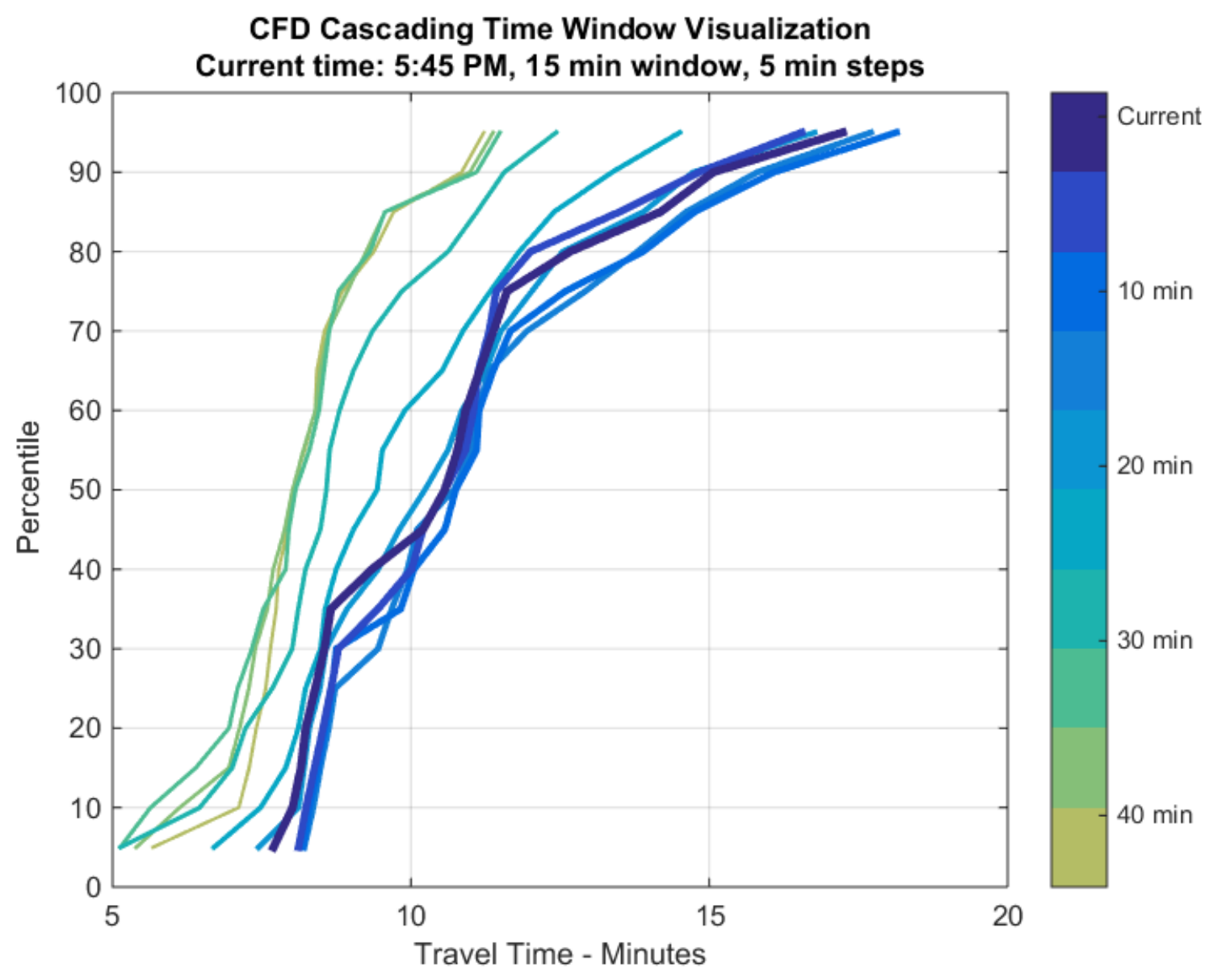

Figure 2.13 Cascading CFD visualization of escalating evening congestion on US-29 

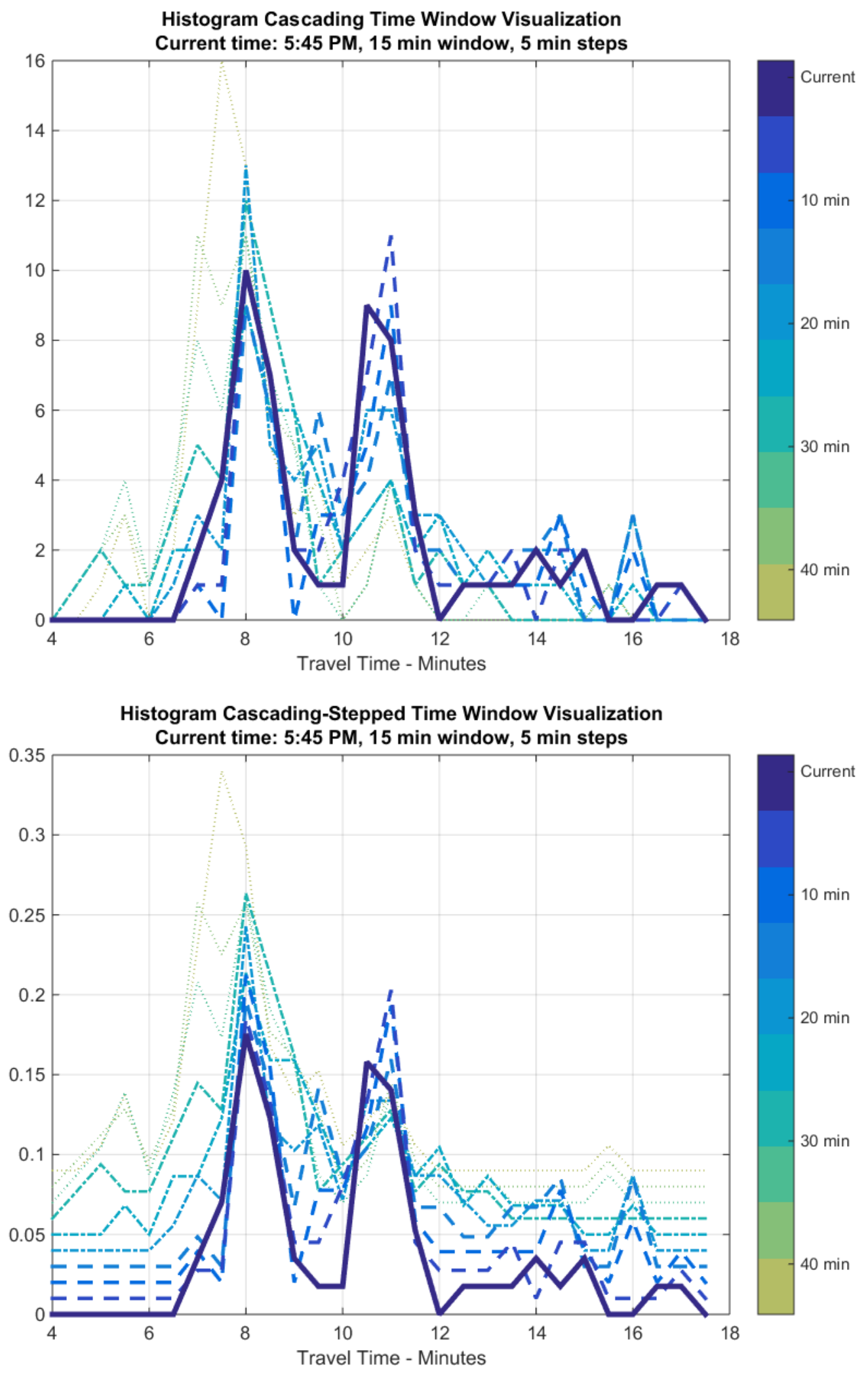

Figure 2.14 Histogram visualizations of escalating evening congestion on US-29 non-stepped (above) and stepped (below) 

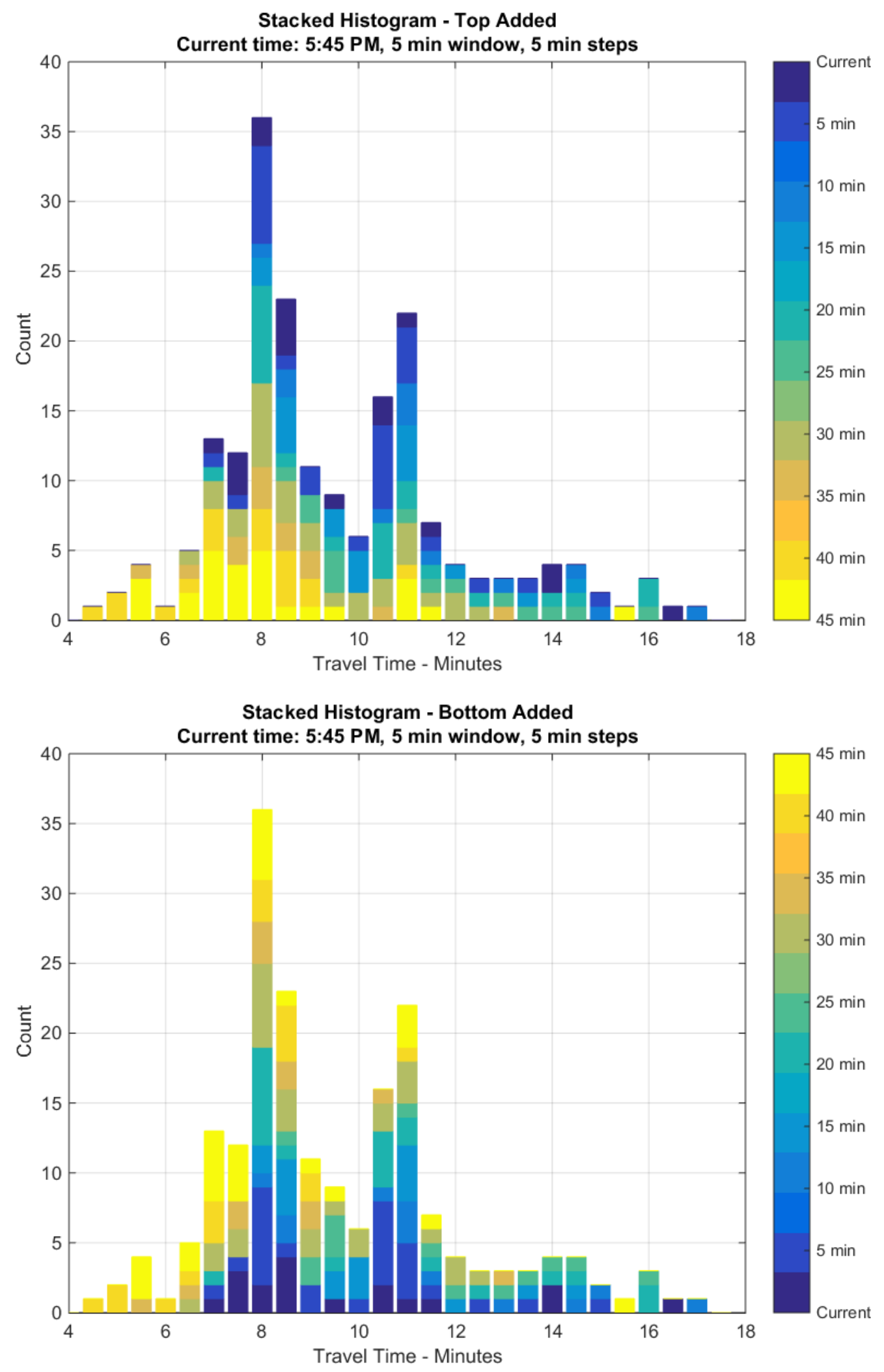

Figure 2.15 Stacked histogram visualizations for US-29: Top-added (above), Bottom-added (below) 
Samples of the visualizations using outsourced probed data for the New Jersey US-1 corridor are shown in the following figures. These visualizations use the outsourced probe data depicted in Figure 2.1 as the base data to construct the various representations.

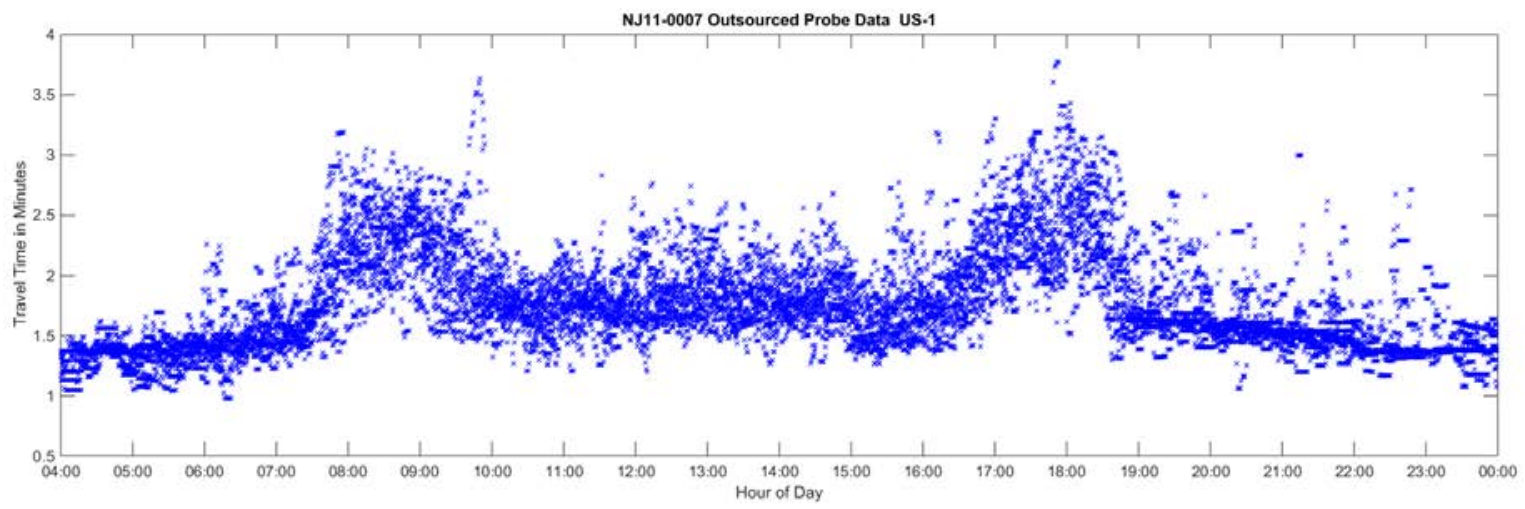

Figure 2.16 Base outsourced probe data for US-1 in New Jersey

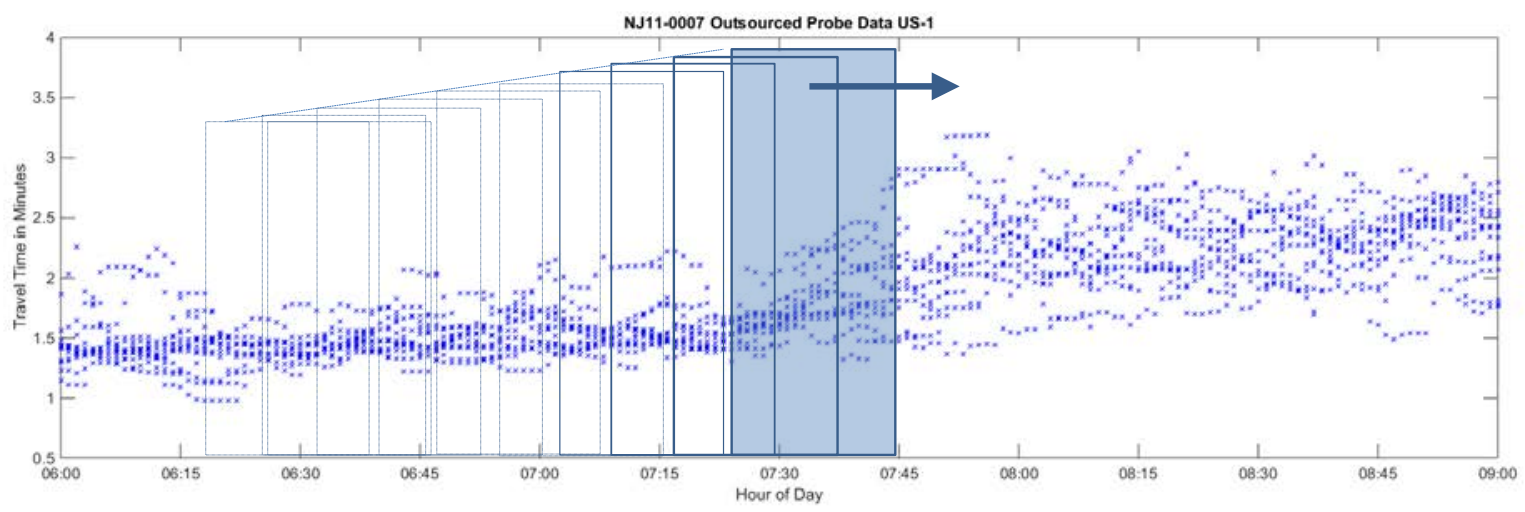

Figure 2.17 View of Outsourced Probe Data from 6 am to 9 am 


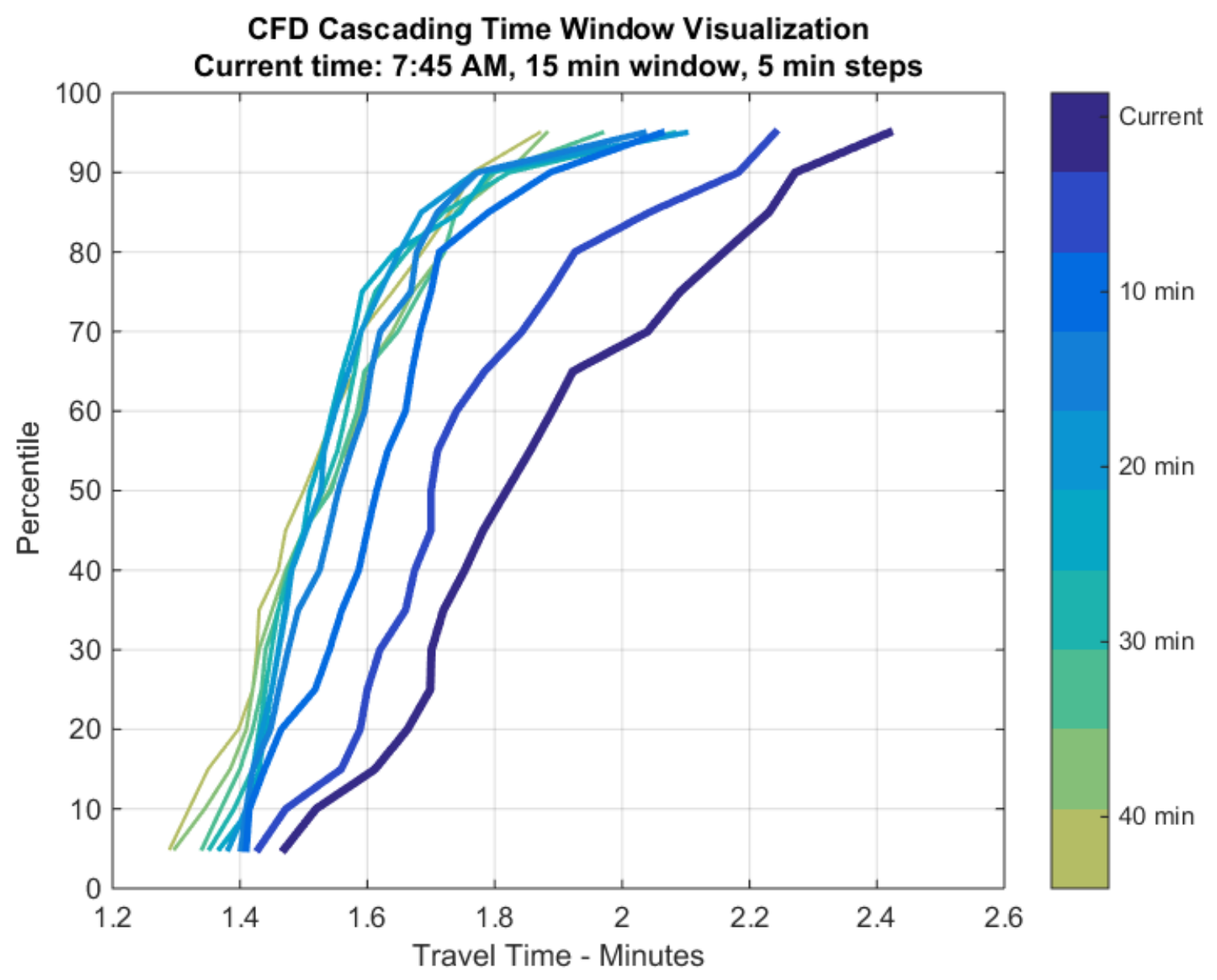

Figure 2.18 Cascading CFD Visualization of Outsourced Probe Data from New Jersey US-1 


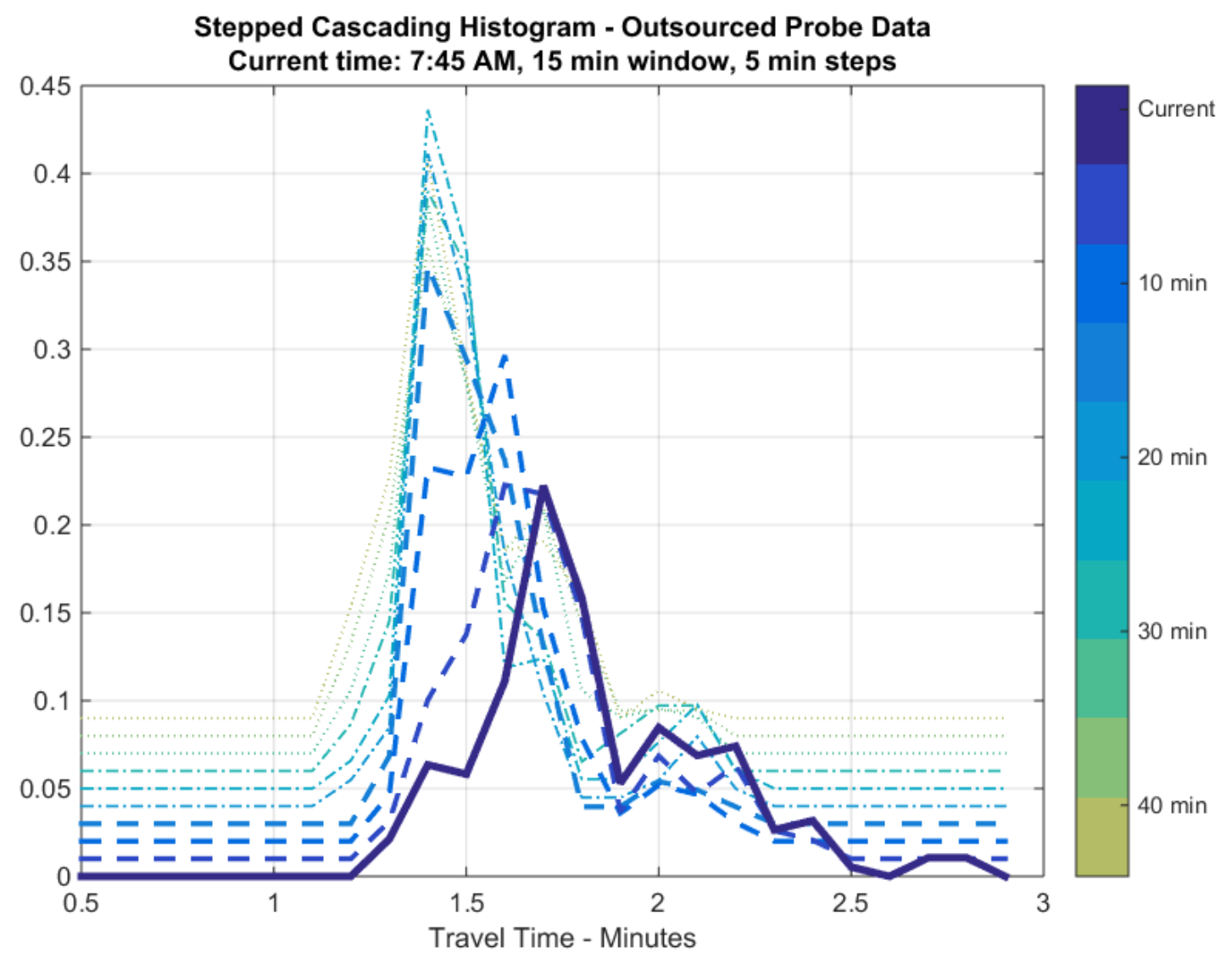

Figure 2.19 Stepped Cascading Histogram of Outsourced Probe Data from New Jersey US-1 
Stacked Histogram - Top Added

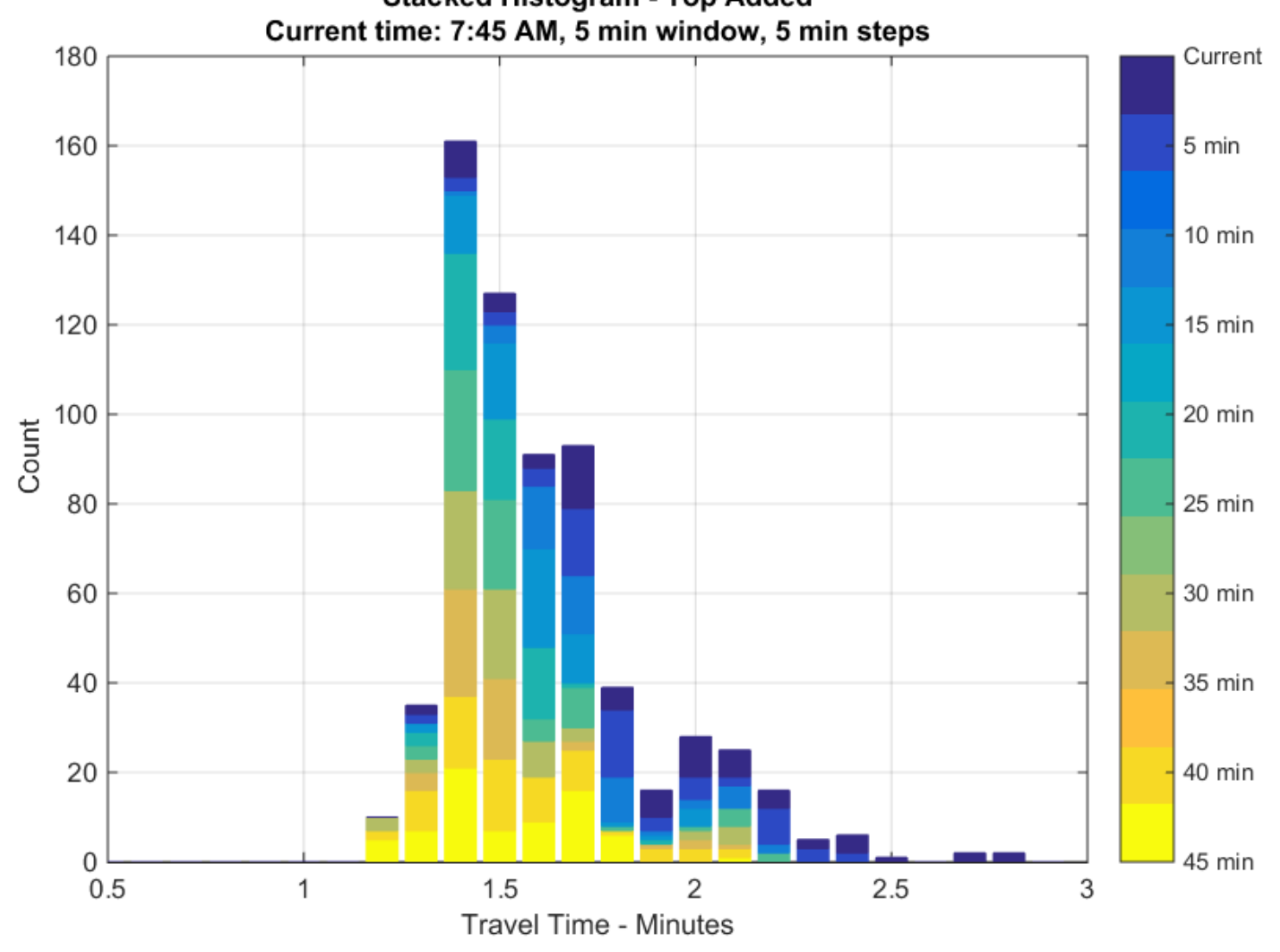

Figure 2.20 Top-Stacked Histogram of Outsourced Probe Data from New Jersey US-1 


\section{Chapter 3: Technical Considerations for Real-Time Arterial Performance Measures}

\subsection{Introduction}

This chapter examines issues related to the sufficiency of the number of travel time samples to support real-time visualization of intersection performance. Chapter 2 introduced proposed visualizations for realtime traffic information on arterial roadways using ample data taken from a two-week overlay chart depicting weekday traffic patterns. As a result of combining two weeks of weekday data, the resulting data density was of a magnitude such that sufficient sample sizes were not a concern. The sufficiency of the number of travel time samples obtained in real-time to support visualizations of intersection performance and the appropriate selection of time averaging windows based on data density and other competing concerns are addressed in this chapter.

In Chapter 2, real-time visualizations were constructed that created a cascading sequence of time window snap shots of traffic performance. For the examples used, the time window was set to 15 minutes, the time step to 5 minutes, and the number of steps to 10 . These parameters provided a visualization that summarized traffic over the previous 45 minutes in a visually appealing format. These parameters were not chosen arbitrarily; rather they were based on arterial signal operations needs with respect to sampling theory principles. Each parameter is discussed below providing guidance for selection appropriate to the situation.

\subsection{Time Window Selection}

The time window is the base unit over which real-time traffic is aggregated. The length of the appropriate time window is governed by three concerns:

1. Interaction with the cycle length of signals along the corridor

2. Minimum sample sizes for statistical significance

3. Time window granularity to support application needs.

These concerns are discussed and addressed independently, and then general guidelines that address all concerns are provided.

\subsubsection{Cycle Length Considerations}

The selection of the minimum time window is fundamentally limited by the cycle length of the single control. As an absolute minimum, the time window should be long enough to capture one complete signal cycle. For instance, if the signal cycle is 90 seconds, any time window less than 90 seconds would capture only a portion of the vehicles that approach a signalized intersection during a single phase. Any time window of substantially less than 90 seconds would risk having no samples for the desired movement, as it may not encompass the phase containing the desired movement.

A practical minimum is a time window that captures three or more cycles. This arises out of concern for partially capturing a green phase of one cycle and thus biasing the aggregate measure of travel time. For example, consider that the cycle time is 90 seconds, and the time window is 1.4 times the cycle time, or 
126 seconds. Successive time windows of 126 seconds will eventually create a situation in which one 126 period will capture the beginning portion of the desired movement during the green phase, while the next 126 periods will capture the remainder of the cycle. When operating near capacity, the front of the queue will experience measurably less travel time than the vehicles deeper in the queue. As a result the sampling period that captures the portion of cycle that services vehicles near the front of the queue will be biased to faster travel times than the succeeding time window that captures portions of the phase that services vehicles deeper in the queue. This situation can result in artificial harmonics introduced into the aggregated data that are a by-product of the selection of the time window with respect to the cycle length. If the cycle length is known, and the time window set to an exact multiple of the cycle length, this effect is cancelled. If the cycle length is unknown, which is commonly the case, or if the cycle length is variable, a time window should be selected that spans three or more complete cycles, minimizing or dampening any possible harmonic effects. As most signal cycles are three minutes or less, a practical minimum of a 10 minute time window satisfies this concern.

\subsubsection{Data Density Considerations}

The minimum time window is also fundamentally limited by the number of independent travel time data samples. The accuracy of the estimated mean for normally distributed data based on the number of data samples is well documented. Minimum sample sizes put bounds on the expected error of the estimated mean travel time as a function of the sampled variance and number of samples. Similar guidelines for characterizing the distribution of bi-modally distributed data, frequently encountered on signalized arterials, are less prevalent.

As opposed to a statistical model, an empirical method was employed to estimate minimum number of data samples to support the CFD visualization in the presence of bi-modal travel time distributions. The Bluetooth re-identification data from weekday overlay plots on US-1 in New Jersey were sequentially resampled to simulate sampling rates of $50,30,20,15,10,8,7,6,5,4,3$, and 2 per five minute period. Figure 3.1 illustrates how this was accomplished for a sample rate of 30 per five minute period. Data from US-1 is re-sampled to 30 data samples per five minute window between 6:00 AM and 9:00 AM on the US-1 test corridor. The re-sampled data is demarked by red circles. For each five minute period 30 samples of the original data were selected at random.

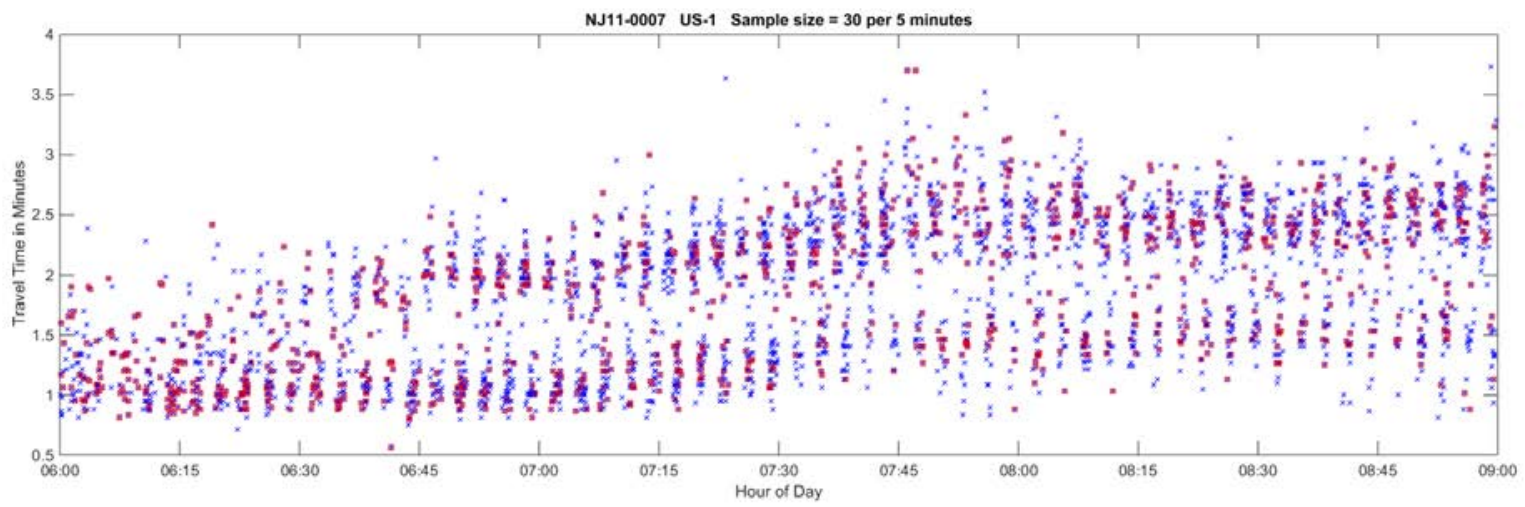

Figure 3.1 Re-sampling of base data to achieve a 30 sample per five minute sampling rate 
The re-sampled data were then used to create cascading CFD plots at each sampling rate that were compared to the baseline cascading CFD in which all available data was used to construct the CFD plot.

Figure 3.2 is one such example for a sampling rate of 30 data points per five minute period. The original cascading CFD using all available data is shown in the upper left. The cascading CFDs created using resampled data are shown in the remaining three graphs. Each graph is generated by randomly selecting (using a pseudo random selection method in software) 30 data points from each five minute interval. This process was repeated for sampling rates of 50, 30, 20, 15, 10, 8, 7, 6, 5, 4, 3, and 2 per five minute period. The corresponding cascading CFDs were produced and reviewed to determine if the same pattern in the baseline cascading CFD was still discernable. The sample using 30 samples per five minute period shown in Figure 3.2 generally reflects the same congestion pattern as the base data over the course of the 45 minute period depicted (15 minute window, 5 minute step, and 10 steps).
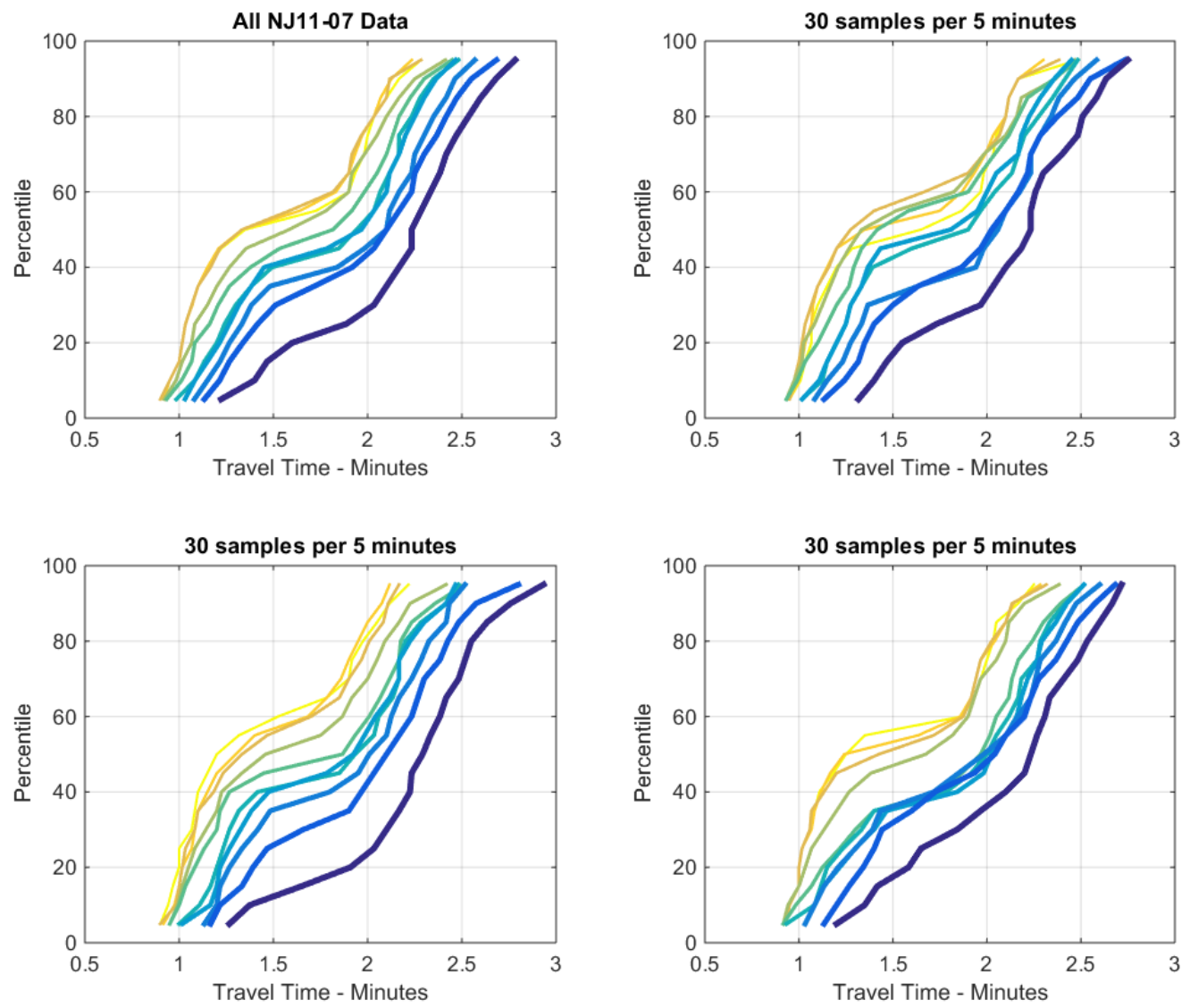

Figure 3.2 Comparison of baseline cascading CFDs (upper left) with CFDs created using 30 samples per 5 minute period (remaining graphs) 
Two additional graphs are displayed from this empirical method. Figure 3.3 reflects a 5 sample per five minute period sampling rate, considered to be the practical minimum to consistently observe differences in travel time of the magnitude equivalent to a cycle length. Although the cascading CFDs are not as refined as the baseline, the trend is discernable, and the sequence of increasing travel times (shifting to the right) is apparent. Figure 3.4 is based on 3 samples per five minute period sampling rate. At this sampling rate the pattern, though arguably present, is not as readily discernible in all three samples.

The resulting guideline created from this experiment is to expand the time window to include at least 15 travel time observations. Note that at 5 samples per five minute window and a 15 minute time window, each CFD line in the CFD plots are based on 15 data points. More is preferable. In extremely sparse data situations an absolute minimum of 10 data points per time window can be used, but the trending pattern may not always be discernable.
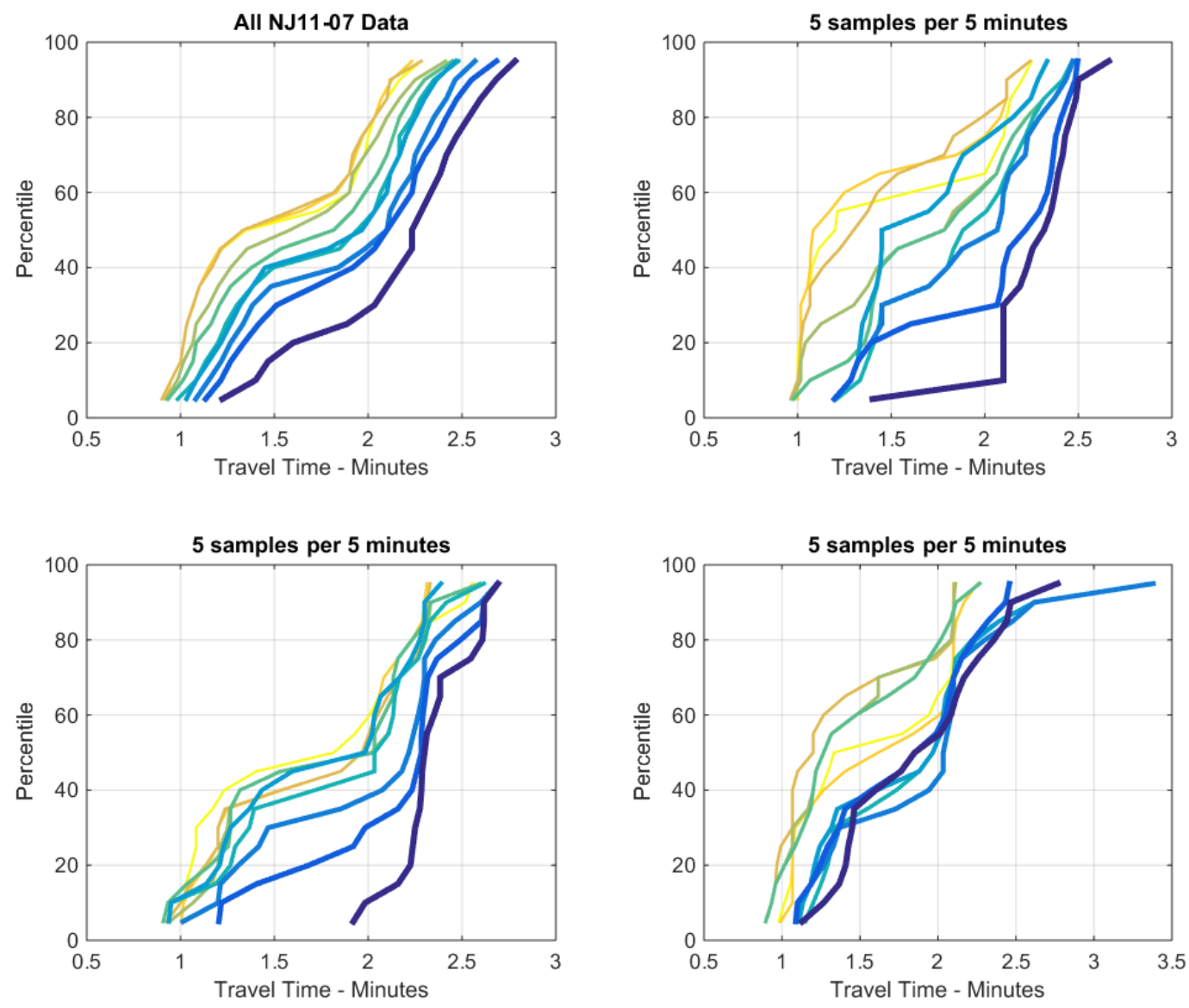

Figure 3.3 Comparison of cascading CFDs created using all data (upper left) and 5 samples per 5 minute period (remaining graphs) 

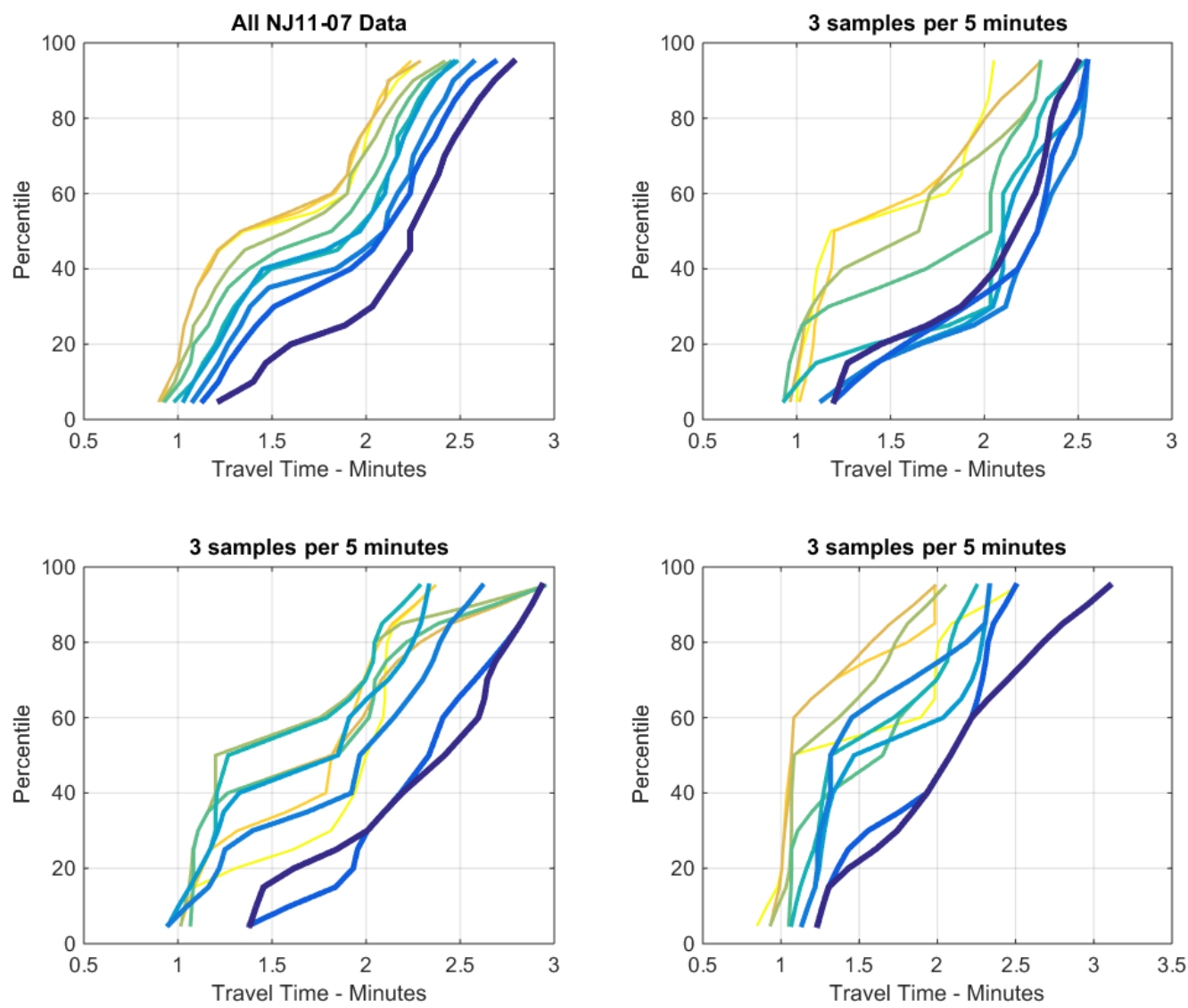

Figure 3.4 Comparison of cascading CFDs created using all data (upper left) and 3 samples per 5 minute period (remaining graphs)

\subsubsection{Application Considerations}

Application concerns should be considered when selecting time window size and other parameters for visualization (and application) of real-time arterial travel time data. Typically such considerations are maximum time horizons for which the data will be useful. Major events that divert traffic onto arterial networks are typically longer than 30 minutes, with most being an hour or more. Correspondingly, time windows that aggregate data to time periods longer than 15 to 20 minutes may not be sufficiently responsive to observe the dynamics of traffic. Traveler information, such as posting travel time on signs, is a common real-time operations application. Updates based on traffic conditions older than 15 to 20 minutes are likely not effective. Traveler information benefits from the most up to date data, so shorter time windows (within the bounds of data integrity) are preferred. 


\subsection{Selection of Step Size}

Recommended step sizes range between $1 / 3$ of the time window size up to the full size of the time window. When the step size is equal to the time window, successive CFD curves (or successive histograms) have no data in common. Each successive time window is independent of the previous. If data density is high, and total time horizon to be monitored is large relative to the time window, the step size can and should approach the size of the time window. For example, if the time horizon is two full hours, and the time window is 15 minutes, a time step equal to the time window may be appropriate.

Step sizes that are fractions (such as $1 / 2$ to $1 / 3$ of the time window), allow successive time windows to share data. In the example with 15 minute time windows, and 5 minute steps, each successive curve in the cascading window shares 10 minutes of data with its neighbor. This overlap acts as a smoothing function, so that the visualization has less-tendency to change abruptly from one time window to the next.
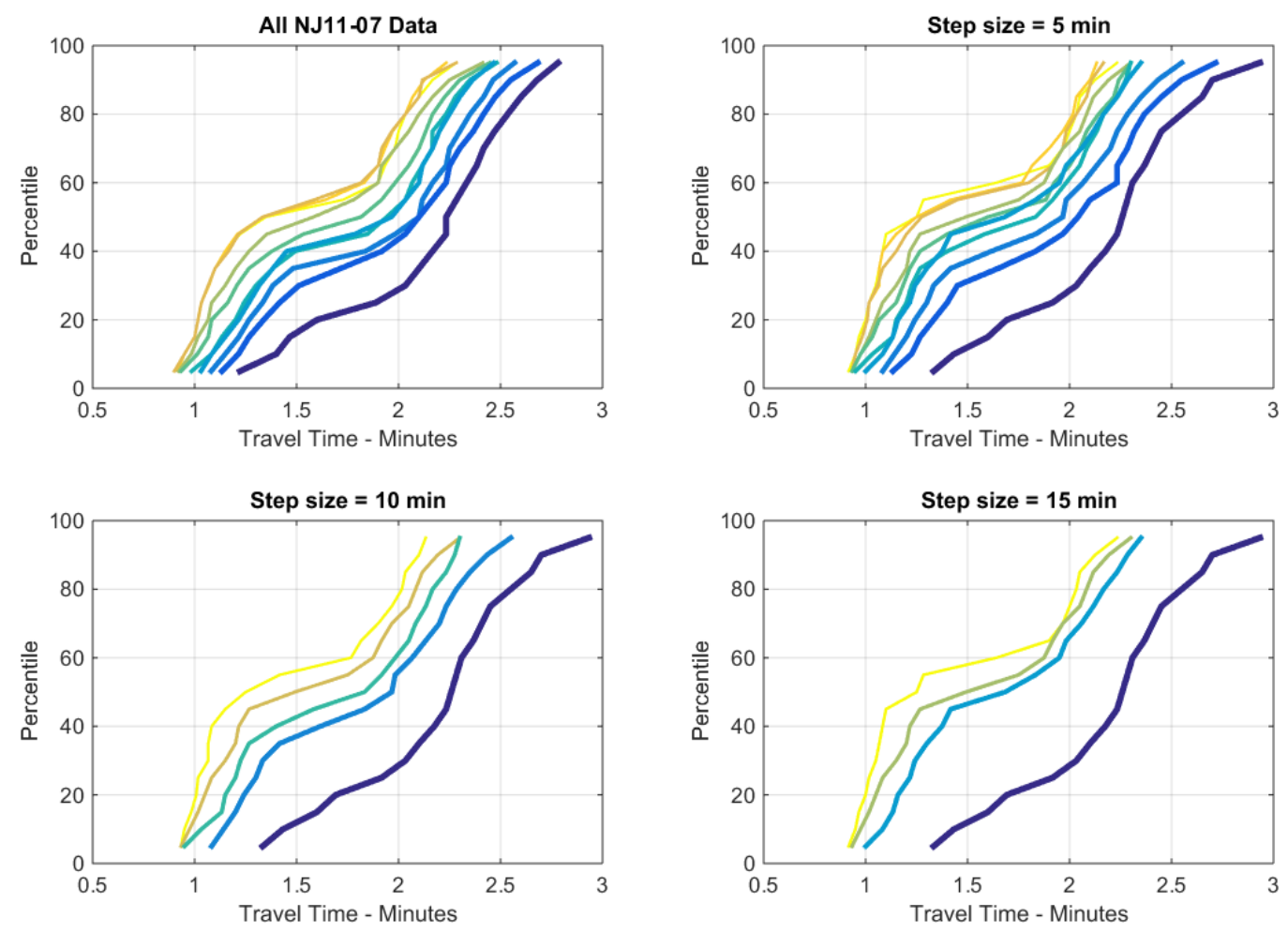

Figure 3.5 Example of 5, 10, and 15 minute time steps with a 15 minute time window.

For practical applications, step sizes are typically determined by the responsivity needs of operations. Updates every 5 minutes or less are typically desired to minimize any latency in the system. Once every five minutes the travel time CFD curve is memorialized, and a new 'current time' curve is initiated. It is unlikely that historical snapshots of less than five minutes would be useful. It is often desirable to display the most current travel time data as it arrives at the traffic management center (TMC). This can be accomplished by constantly updating the most current time window (most current CFD curve) with travel time data as it arrives. Using the example of a 15 minute time window with 5 minute steps, once a fiveminute epoch is reached, the 15 minute time-window just completed is archived, and the next 15 minute 
time-window reflecting the most current conditions is initiated with data from the last 10 minutes. As time progresses and data are received, the most recent CFD curve is updated with each new travel time datum. Once the next five minute epoch is passed (completing the current 15 minute time window), the current 15 minute time window is again archived, and the next time-window is initialized with the most recent 10 minutes of data, and the process repeated.

\subsection{Number of Steps}

The total number of steps along with the step size determines the history portrayed in the visualizations and the density of lines. An alternate method is to designate the total time to display, and calculate the number of steps needed to span that history. The density of lines in either a cascading CFD or cascading histogram is critical, as it strikes a balance between sufficient detail and excessive complexity. In the example, 10 steps covering 45 minutes yielded acceptable results, with 10 distinct lines displayed on the CFDs. Any more than 10, and the display may become too busy or cluttered. Any less than 5, and the detail, or sense of time sequencing may be compromised (see Figure 3.5).

\subsection{Implementation Considerations}

In summary, guidelines for implementing cascading visualizations include, with respect to windowing parameters:

- Choose a time-window appropriate for the data, typically 10 to 20 minutes

- Step size should be chosen appropriate to the total time horizon, such that the total number of steps is between 5 and 10 .

- Expand the time-window to the maximum time if the number of samples per time period is lean. Preferred minimum sample size per time window is 15 data samples.

- Flag or denote time windows if the total number of data samples falls to less than 10

- Sample size may be incorporated into the visualization as secondary information

Note that the time windowing parameters were introduced and discussed based on the assumption of uniform time windows and equal time steps. Hybrid approaches may be incorporated in which the most recent data uses smaller time windows and steps, and older data (such as 30 minutes to 2 hours ago) use larger time windows and steps. This provides the ability to use higher granularity and more steps (or lines) for more recent data, and larger aggregation windows and less steps (or lines) for older epochs. For example, the first 30 minutes of data may use a 15 minute time window with 5 minute steps, then once the data ages to 30 minutes old, the visualization may transition to a 15 minute step. In this scenario, 10 steps could be used to display a two hour time horizon.

Other implementation considerations:

- Having user selectable color schemes is important, as many visual impairments impact the contrast between select color pairs. 


\section{Chapter 4: Real-time High Resolution Controller Data}

The report entitled "Performance Measures of Interrupted-Flow Roadways using Re-Identification and Signal Controller Data” developed several performance measures from high-resolution controller data to aid in the management and optimization of signal-controlled corridors. Two of these measures have potential for real-time situational awareness during incidents. High-resolution controller data have two distinguishing features from re-identification data:

- High-resolution controller data directly reflects the performance of the individual signal, and not the experience of the traveler. It can provide feedback at a specific location about how efficiently an intersection is functioning for all traffic (not just the diverted traffic). In contrast, reidentification data reflects the experience of travelers (based on travel time) through a corridor which may encompass several signalized intersections.

- Whereas the responsiveness of re-identification data is inherently limited by the completion time of a trip between sensors (a vehicle must be re-identified downstream before any information about its travel time is known), high-resolution controller data is governed primarily by its cycle length. Information on its ability to service traffic is available (if conveyed in real-time) on a cycle by cycle basis.

Conveying high-resolution controller data in real-time is not typical practice, as it is for re-identification data. This chapter comments on useful information that could be obtained, provided that real-time data is conveyed and processed.

\subsection{Real-time GOR and ROR to Detecting Queuing}

Two performance measures based on occupancy data from the stop bar detector are applicable to realtime monitoring. These are the Red Occupancy Ratio (ROR) and the Green Occupancy Ratio (GOR).

The ROR is the proportion of time during the first five seconds of red (immediately after the signal transition from yellow to red for the associated movement) that a stop bar detector is occupied. The GOR is the proportion of time during green during which the stop bar detector is occupied. High GOR indicates that the phase was utilized by vehicles during most or all of the green, while high ROR indicates that one or more vehicles were stopped at the signal after the end of service on the movement. A combined high GOR and high ROR in the same cycle (for a green interval and the immediately following 5-second red interval) is an indicator that the amount of traffic demand exceeded the capacity during that cycle. Repeated occurrence of high occupancy ratios on multiple successive cycles is an indication of oversaturation, indicating the queue is not being cleared for that approach. The rule of thumb is that if ROR and GOR remain high for three successive cycles, then queuing conditions resulting from split failure are highly likely. A combination of high ROR and high GOR (>0.80) on successive cycles flags oversaturated conditions, likely split failures, and likely growth of a queue.

ROR and GOR can be used in real-time to provide feedback on intersection conditions, not only for the mainline traffic, but also for side streets as a result of redirected traffic, or signal plans put into effect to handle redirected traffic. If signal timing is altered to better handle diverted traffic, normal traffic on side streets may be unduly affected. Figure 4.1 is a sample visualization of real-time monitoring that depicts 
the combination of ROR and GOR on each approach to the intersection of the US-40 (Baltimore National Pike) with St. John's Lane, the first signalized intersection to the east of the interchange of US-29 and US-40, also the first intersection on the southern diversion path for the scenario described in Chapter 1. The rectangles on each approach reflect the number of consecutive previous cycles in which the ROR and GOR both exceeded $80 \%$. If that number climbs to three or above, the background of the rectangle is color coded to draw attention to a likely escalating queue.

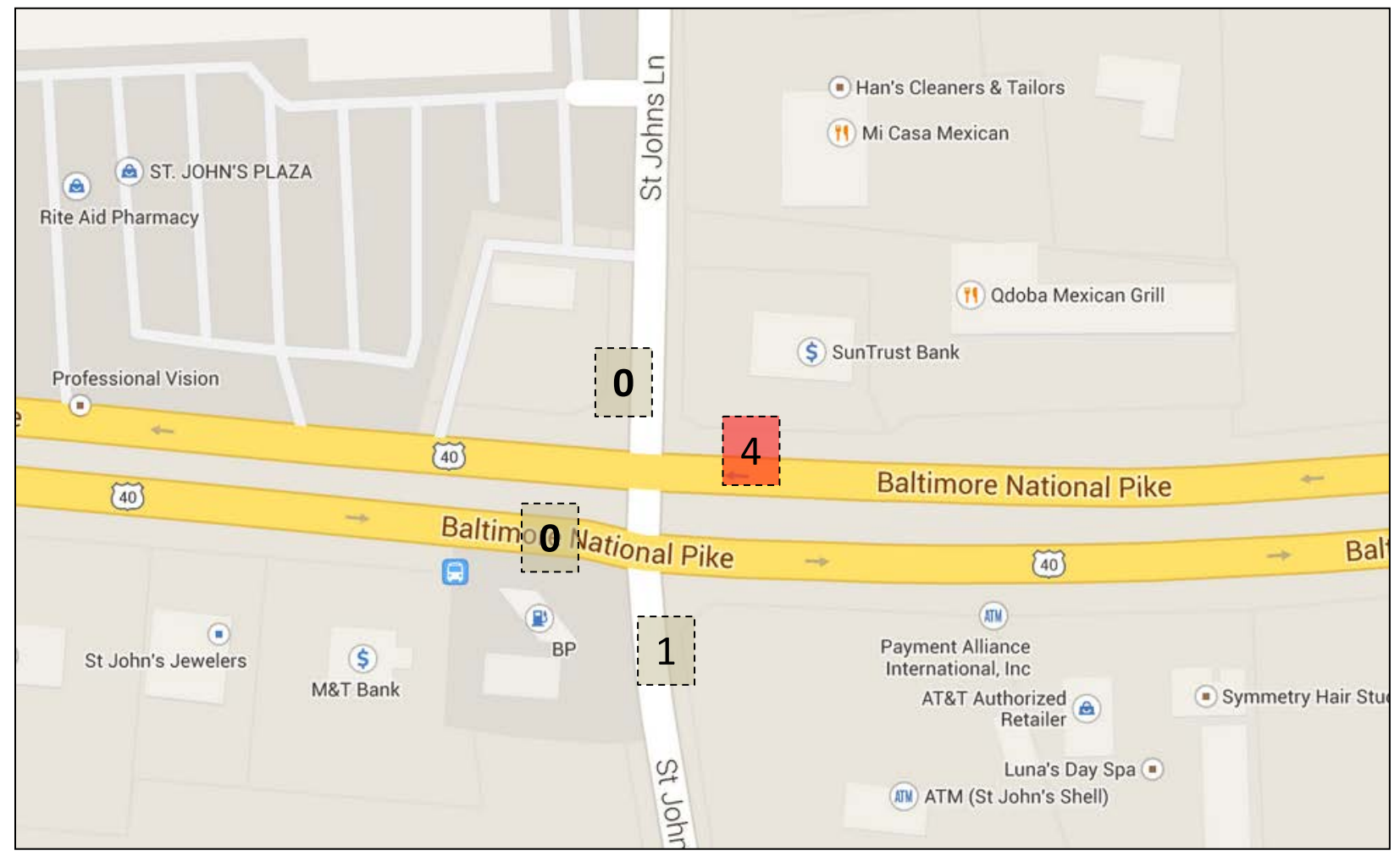

Figure 4.1 Sample visualization of the signal

Similarly, if an alternate signal time plan were instituted on the US-40 corridor to provide greater capacity to the westbound through movement as a result of the diversion, side streets may become starved for adequate green time to completely service demand. So after the signal timing goes into effect, it is equally essential to monitor side streets to detect adverse consequences. Such a situation is shown in Figure 4.2, in which northbound traffic on St. John's lane is starved for green when after signal timing has alleviated the westbound queuing that was developing as a result of the traffic diversion from the freeway.

Note both Figure 4.1 and Figure 4.2 reflect an aggregated view across all lanes of traffic on an approach, including the left and right turns and the through movements. Intersection specific layout may need to be considered with respect to visualizations, and balanced against considerations to minimize complexity of display. 


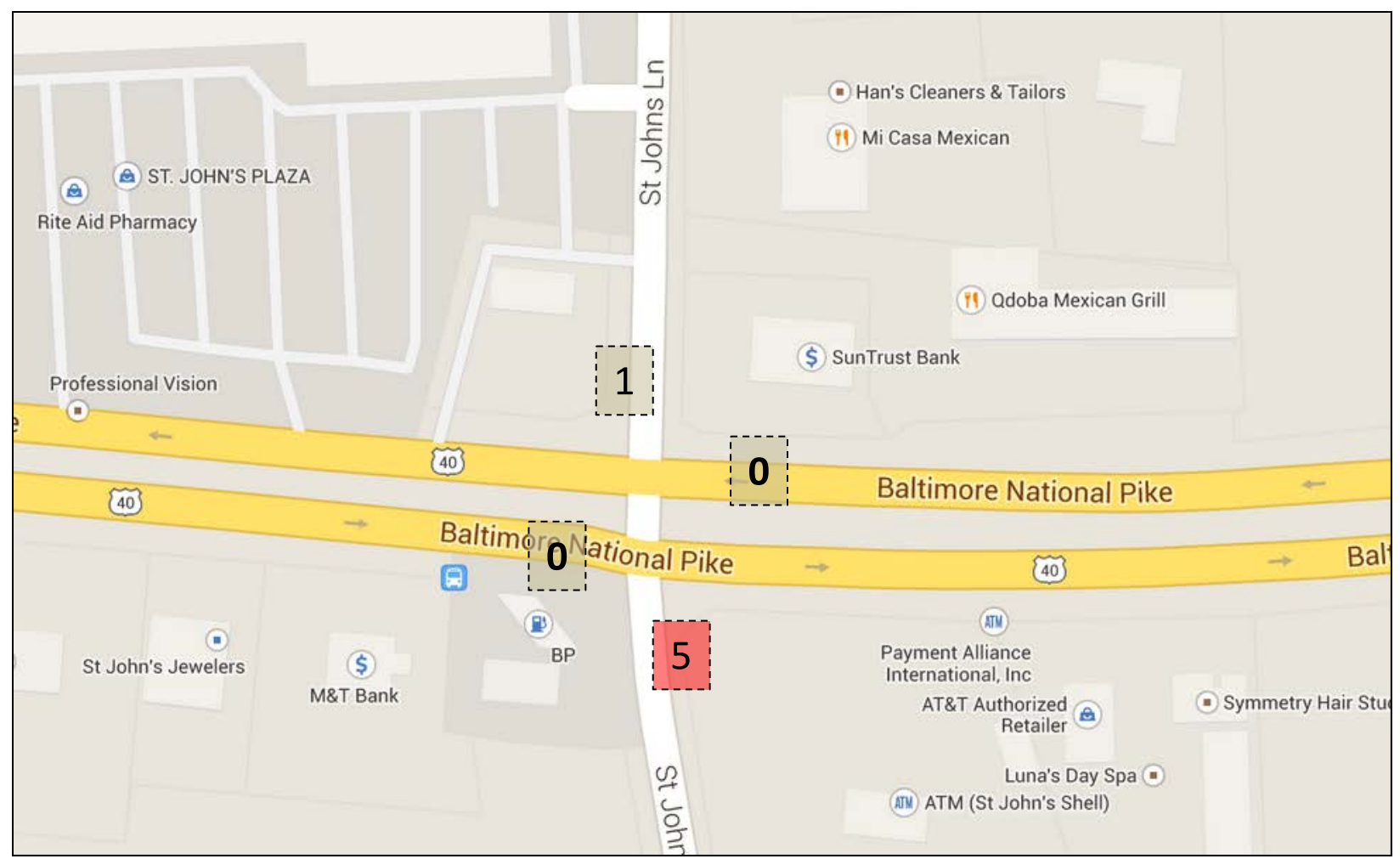

Figure 4.2 Application of ROR and GOR in real-time to detect side street disruptions due to signal timing changes.

\subsection{Real-Time Volume Estimates}

Volume estimates from upstream sensors, the same sensor as utilized for Purdue Coordination Diagrams, may provide additional information for operations. However, there is no precedence or practice to leverage real-time volume data based on previous experience on freeways within TMCs. Inductive loops, side fire radar, and other freeway sensors are typically used for speed estimates on freeways. Volume estimates derived from upstream sensors provide little value without corresponding knowledge of nominal conditions (nominal demand) for the time of day and day of week. In contrast, the ROR and GOR detection schemes in Figure 4.1 require no previous knowledge of nominal intersection conditions (other than the expectation that split-failure and over-saturation are not nominal conditions) in order to be effective. Also, once queuing begins, and progresses past the approach sensor, the measured volume will reflect the intersection capacity, rather than demand. 


\section{Report Sponsor}

The "Small Business Innovation Development Act of 1982" (Pub. L. No. 97-219), along with reauthorizing legislation (Pub. L. No. 99-443 and Pub. L. No. 102-564, the "Small Business Research and Development Enhancement Act of 1992"), seeks to encourage the initiative of the private sector and to use small business effectively to meet federal research and development objectives. To comply with statutory obligations of the Act, the U.S. Department of Transportation established the Small Business Innovation Research (SBIR) Program, which conforms to the guidelines and regulations provided by the Small Business Administration. Annually, small businesses are solicited to submit innovative research proposals that address the high-priority requirements of the U.S. Department of Transportation and that have potential for commercialization.

This report was developed through a partnership between Traffax, Inc., and Purdue University with funding from a Phase III SBIR contract (DTFH6114C00035) with the Federal Highway Administration. The project, entitled "Sensor Fusion and MOE Development for Off-Line Traffic Analysis of Real Time Data," created and refined methods and tools for the characterization of performance along arterial corridors.

\section{Publication}

This report is part of a series of reports published in collaboration with USDOT, Traffax, Inc., and Purdue University. The full report series is available for download at http://docs.lib.purdue .edu/apmtp/.

\section{Open Access and Collaboration with Purdue University}

The Indiana legislature established the Joint Highway Research Project in 1937. In 1997, this collaborative venture between the Indiana Department of Transportation and Purdue University was renamed as the Joint Transportation Research Program (JTRP) to reflect state and national efforts to integrate the management and operation of various transportation modes. Since 1937, the JTRP program has published over 1,600 technical reports. In 2010, the JTRP partnered with the Purdue University Libraries to incorporate these technical reports in the University's open access digital repository and to develop production processes for rapidly disseminating new research reports via this repository. Affiliated publications have also recently been added to the collection. As of 2017, the JTRP collection had over 1.5 million downloads, with some particularly popular reports having over 20,000 downloads. 